\title{
Structural health monitoring in changing operational conditions using tranmissibility
} measurements

\author{
Christof Devriendt, Flavio Presezniak, Gert De Sitter, Katy Vanbrabant, Tim De Troyer, \\ Steve Vanlanduit and Patrick Guillaume* \\ Department of Mechanical Engineering, Acoustics and Vibration Research Group, Vrije Universiteit Brussel, \\ Brussel, Belgium
}

\begin{abstract}
This article uses frequency domain transmissibility functions for detecting and locating damage in operational conditions. In recent articles numerical and experimental examples were presented and the possibility to use the transmissibility concept for damage detection seemed quite promising. In the work discussed so far, it was assumed that the operational conditions were constant, the structure was excited by a single input in a fixed location. Transmissibility functions, defined as a simple ratio between two measured responses, do depend on the amplitudes or locations of the operational forces. The current techniques fail in the case of changing operational conditions. A suitable operational damage detection method should however be able to detect damage in a very early stage even in the case of changing operational conditions. It will be demonstrated in this paper that, by using only a small frequency band around the resonance frequencies of the structure, the existing methods can still be used in a more robust way. The idea is based on the specific property that the transmissibility functions become independent of the loading condition in the system poles. A numerical and experimental validation will be given.
\end{abstract}

Keywords: Operational conditions, health monitoring, transmissibility

\section{Introduction}

Structural health monitoring is still an ongoing and challenging research. A lot of work has been published in this field and an extensive overview can be found in [1]. More recently Farrar et al. (2001) reviewed the state-of-the-art in vibration-based damage detection and discussed it in [2].

The goal of structural health monitoring is to detect damage before it becomes critical for the structure's function and integrity. Damage alters the dynamic characteristics of a structure, namely its natural frequencies, modal damping and modes of vibrations. Therefore several techniques based on experimental modal analysis have been developed in the last years. Although the identification of modal parameters have become very reliable; there is still a general preference for methods that make direct use of raw data like Frequency-Response-Functions [3,4]. One of the major drawbacks in the methods using Frequency-Response-Functions is the necessity to know the forces that are applied to the structure. In case of structures in operation this is often impossible, therefore techniques that do not need the specific knowledge of the applied forces would in many cases be more appropriate for structural health monitoring.

This article will use transmissibility functions for detecting and locating damage in operational conditions. Transmissibility functions are obtained by taking the ratio between two measured responses; therefore no knowledge of the applied forces is needed. In previous work $[5,6]$ it was shown that the use of transmissibility func-

\footnotetext{
*Corresponding author. E-mail: avrg@vub.ac.be; www.avrg.vub.ac.be.
} 
tions for damage detection seems promising. Techniques based on comparing two sets of transmissibility functions of the undamaged and damaged structure have been developed.

Papers so far have focused on situations where the structure was excited by a single input in a fixed location. In other words the input location of the applied force was the same before and after damage occurred. Transmissibility functions, defined as a simple ratio between two measured responses, do however depend on the amplitudes or locations of the operational forces. In many operational conditions the inputs to the structure can vary with time in magnitude and location, e.g. the wind load on a bridge. Therefore it is reasonable to expect that a change in the location of the applied force would imply a noticeable change in the transmissibility functions so it is not unthinkable that false damage detection might occur in these situations.

The proposed solution in this paper is based on an interesting property of transmissibility functions, that was recently shown in [7]. It was proven that the limit value of the transmissibility functions in the poles of the system converge to a unique value and become independent of the operational forces. Taking in mind this property it will be demonstrated in this paper that, by using only a small frequency band around the resonance frequencies of the structure, the existing methods can still be used in changing operational loading conditions.

In the next paragraphs it will be shown how current techniques fail to correctly identify the damage location due to a change in the location of the applied force. Next it will be shown how a careful choice of the selected frequency band can avoid false damage notifications and make the current techniques based on transmissibility measurements more robust. A numerical and experimental example using a cantilever beam will be presented in order to illustrate the method.

\section{Numerical model}

The method will first be demonstrated on a finite element model of a steel cantilever beam given in Fig. 1 with dimensions: $1 \mathrm{~m} \times 0.01 \mathrm{~m} \times 0.01 \mathrm{~m}$.

A finite element model is created, using the commercially available software COMSOL. In COMSOL, a default mesh is applied and the responses are calculated, using a direct solver. The model allows us to apply a unit-point force in 10 points and to measure the responses in all these points. The 10 points are equally distributed over the full length of the beam. Damage is applied to the structure by creating a cut of width $0.004 \mathrm{~m}$ and depth $0.0034 \mathrm{~m}$ between point 7 and point 8 (Fig. 2).

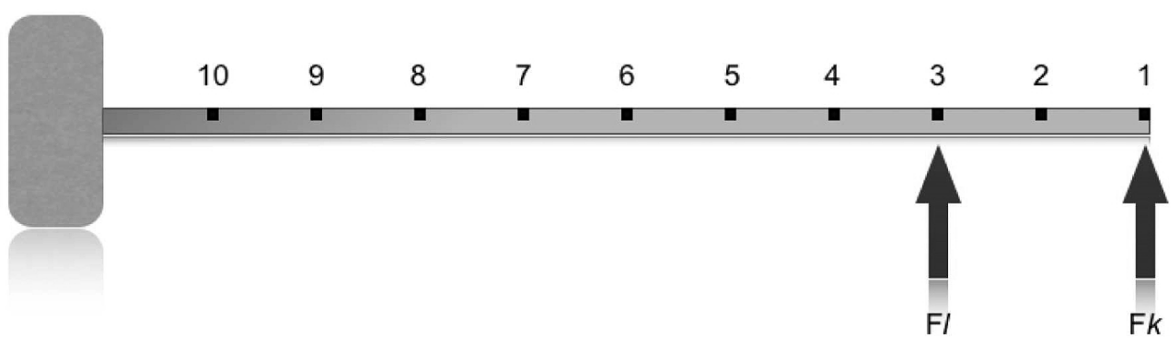

Fig. 1. Cantilever beam.

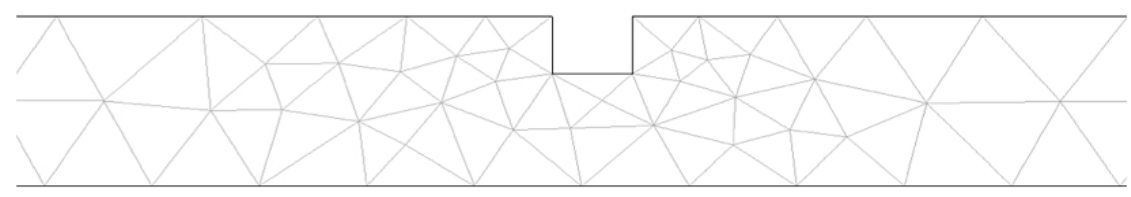

Fig. 2. Zoom on cut in finite element model. 

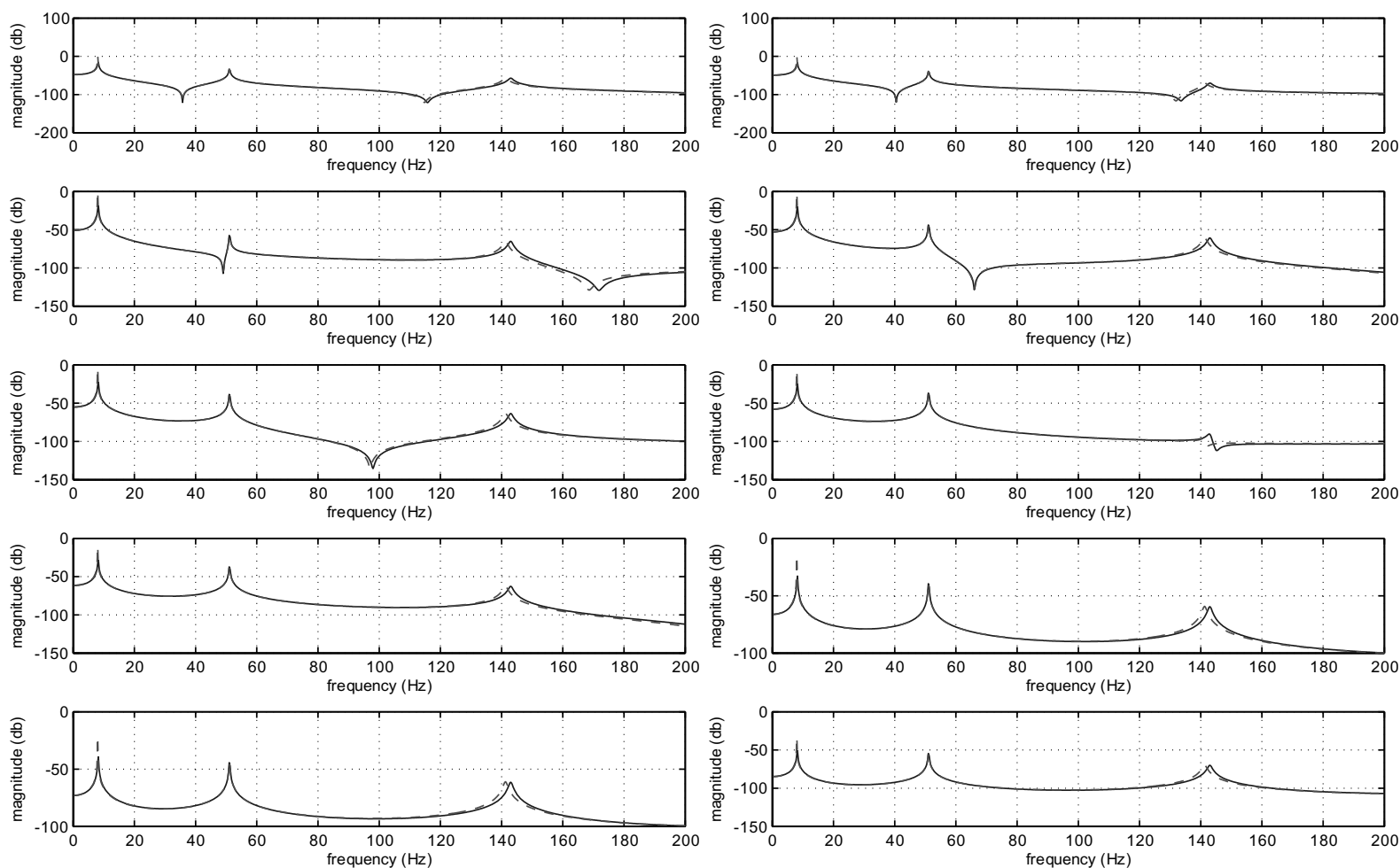

Fig. 3. Response functions for undamaged (full line) and damaged (dashed line) case, with response in DOF 1 (top left), DOF 2 (top right) ... DOF 10 (bottom right).
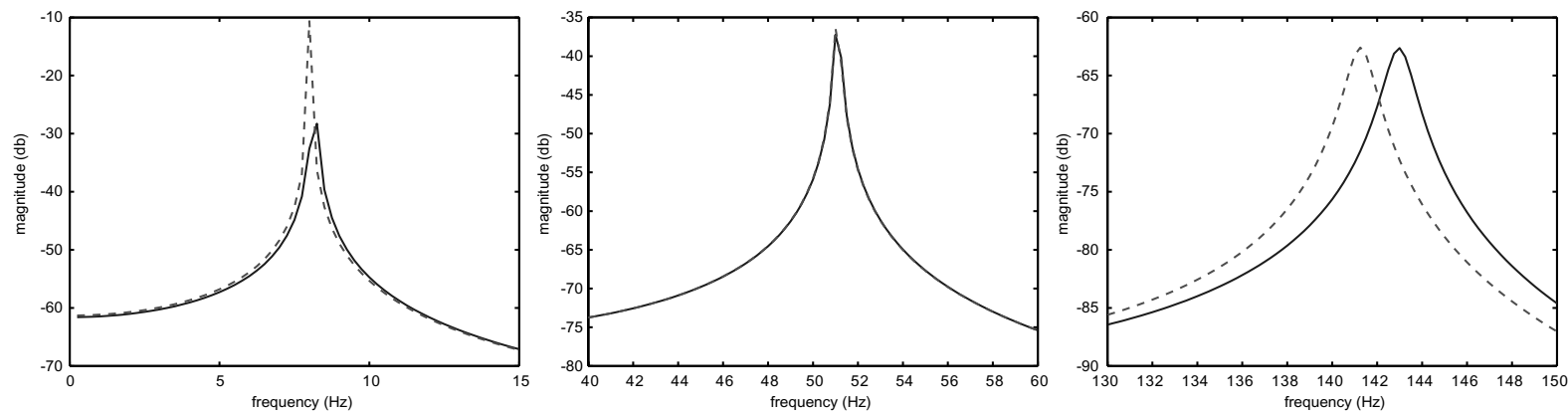

Fig. 4. Zoom on response functions of point 7 around resonance frequencies for undamaged (full line) and damaged (dashed line) case.

In both the undamaged and the damaged case two different loading conditions are considered. The first loading condition $k$ consists of a unit harmonic point force, $F_{k}$, applied in the vertical direction in location 1 . The second loading condition $l$ is a unit harmonic point force, $F_{l}$, applied in location 3 (Fig. 1). Both experiments are performed in the frequency range of $0.1 \mathrm{~Hz}$ to $200 \mathrm{~Hz}$.

Figure 3 shows all the response functions measured for the undamaged and damaged structure for the first loading condition. A small shift between both functions can be noticed. In the frequency range of interest 3 resonance frequencies of the beam are present. The first resonance frequency of the undamaged structure is at $8.25 \mathrm{~Hz}$, the second at $51 \mathrm{~Hz}$ and the third at $143 \mathrm{~Hz}$.

The damage and shift of resonance frequency is most obvious at the first and third mode (Fig. 4). The second mode is almost not influenced by the damage. This can be explained by the fact that the damage is located near a node of the second mode of the cantilever beam. 

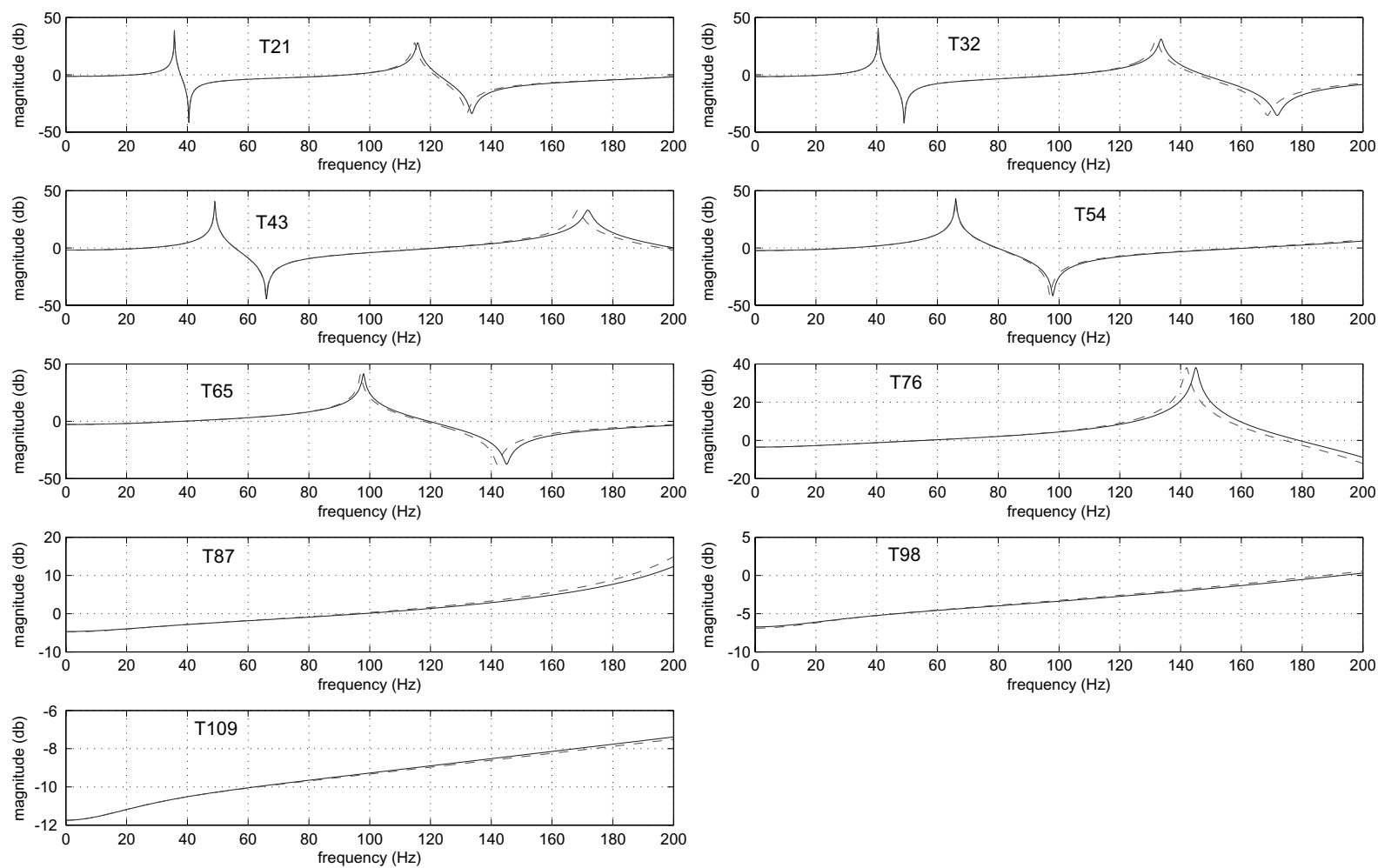

Fig. 5. Transmissibility functions for undamaged (full line) and damaged (dashed line) case for the first loading condition, between responses at DOF 1 and DOF 2 (top left), DOF 2 and DOF 3 (top right) ... DOF 9 and DOF 10 (bottom left).

\section{Transmissibility functions}

In this paper transmissibility functions will be used as primary data to perform the structural health monitoring and detect and locate the damage.

Transmissibility functions are obtained by taking the ratio of two response spectra. These transmissibility functions can, in a similar way as FRFs, be estimated in a non-parametric preprocessing step. The measured vibration responses are divided into different data blocks in order to average and estimate the transmissibility functions.

By assuming a single force $F_{k}(s)$ that is located in, say, the input degree of freedom (DOF) $k$, the transmissibility is given by

$$
T_{i j(k)}(s)=\frac{X_{i k}(s)}{X_{j k}(s)}=\frac{H_{i k}(s) F_{k}(s)}{H_{j k}(s) F_{k}(s)}
$$

with $H_{i k}(s)$ and $H_{j k}(s)$ elements of the transfer function matrix of the structure and $X_{i k}(s)$ and $X_{j k}(s)$ the responses measured at DOF $i$ and DOF $j$ ( $s$ is the Laplace domain variable).

Note that transmissibility functions as defined in Eq. (1) depend on the location of the input DOF $k$ of the unknown force.

In the same way we can define the transmissibility function between the same pair of responses after damage occurred to the structure

$$
T_{i j(k)}^{D}(s)=\frac{X_{i k}^{D}(s)}{X_{j k}^{D}(s)}
$$

Since the dynamic responses of structures change in the case damage occurs it is natural to expect that also the transmissibility functions will change if damage occurs. This can be observed in Fig. 5. 
In order to locate the damage the transmissibility functions will be obtained by taking the ratio between closely spaced neighbor responses. This relation can be designated as transmissibility between two consecutive locations, $i$ and $j=i+1$. Using transmissibility functions that are a ratio of two vibration responses across small sections of the structure will allow us to locate the damage. A change in the transmissibility functions represent a change in the structural properties, and it is presumed that the transmissibility function with the greatest change from the healthy structure indicates the damage location.

\section{Damage indicators}

The first step in order to detect and locate damage with transmissibility functions is the selection of a damage indicator. Maia et al. [5] have already posed the following indicator based on the differences in amplitude between the transmissibility functions of the healthy and damaged structure.

$$
D I_{i j(k)}=\sum_{\omega}\left|T_{i j(k)}^{D}(\omega)-T_{i j(k)}(\omega)\right|=\sum_{\omega}\left|\frac{X_{i k}^{D}(\omega)}{X_{j k}^{D}(\omega)}-\frac{X_{i k}(\omega)}{X_{j k}(\omega)}\right|
$$

By using a uniform frequency weighting the above damage indicator stores one number per transmissibility function. Therefore if we compute the transmissibility functions between consecutive locations, defined on the damaged structure and subtract the same transmissibility functions of the undamaged structure, the damage location should come up with the biggest value of the indicator.

Johnson and Adams [6] have also posed a similar indicator:

$$
D I_{i j(k)}=\left|\sum_{\omega}\left(1-\frac{T_{i j(k)}^{D}(\omega)}{T_{i j(k)}(\omega)}\right)\right|=\left|\sum_{\omega}\left(1-\frac{\frac{X_{i k}^{D}(\omega)}{X_{j k}^{D}(\omega)}}{\frac{X_{i k}(\omega)}{X_{j k}(\omega)}}\right)\right|
$$

Note, neither indicator is independent of the order of the response ratios.

$$
D I_{i j(k)} \neq D I_{j i(k)}
$$

Therefore Johnson [8] improved the method by using the absolute value of the common logarithm of the transmissibility functions. He proposed to use a temporary variable called the transmissibility power Eq. (6), which is independent of the order of the output responses in the transmissibility ratio.

$$
T P_{i j(k)}=\left|\log \left(T_{i j(k)}(\omega)\right)\right|=\left|\log \left(\frac{X_{i k}(\omega)}{X_{j k}(\omega)}\right)\right|
$$

Johnson proposed the following damage indicator, hereafter called the damage feature.

$$
D I_{i j(k)}=\left|\sum_{\omega}\left(\frac{T P_{i j(k)}^{D}(\omega)-T P_{i j(k)}(\omega)}{T P_{i j(k)}(\omega)}\right)\right|
$$

It was shown by Johnson [8] that the proposed damage indicator Eq. (7) is able to correctly diagnose linear and non linear types of damage and able to provide good and consistent results in terms of detection, location, and quantification of the damage under the assumption of a single force exciting the structure at a constant location on the structure. In this paper the damage feature as defined in Eq. (7) will be used.

We will now calculate the damage feature and store one number per pair of transmissibility functions between 2 consecutive locations on the healthy and damaged structure. The damage location should come up with the biggest value of the damage feature. We will randomly choose 3 different frequency bands, respectively the full band of 0-200 Hz, a band between $60 \mathrm{~Hz}$ and $120 \mathrm{~Hz}$ and a band between $10 \mathrm{~Hz}$ and $40 \mathrm{~Hz}$ (Fig. 6).

The damage is most obvious in the first 2 frequency bands. The damage can correctly be located at transmissibility pair 7, meaning between DOF 7 and DOF 8 . Also in the frequency band $10-40 \mathrm{~Hz}$ one can still correctly locate the damage in the neighborhood of location 7 , but some other high values of the damage feature can be found at nearby locations. Also in the 2 other bands some non-zero values of the damage feature can be found at other locations than 

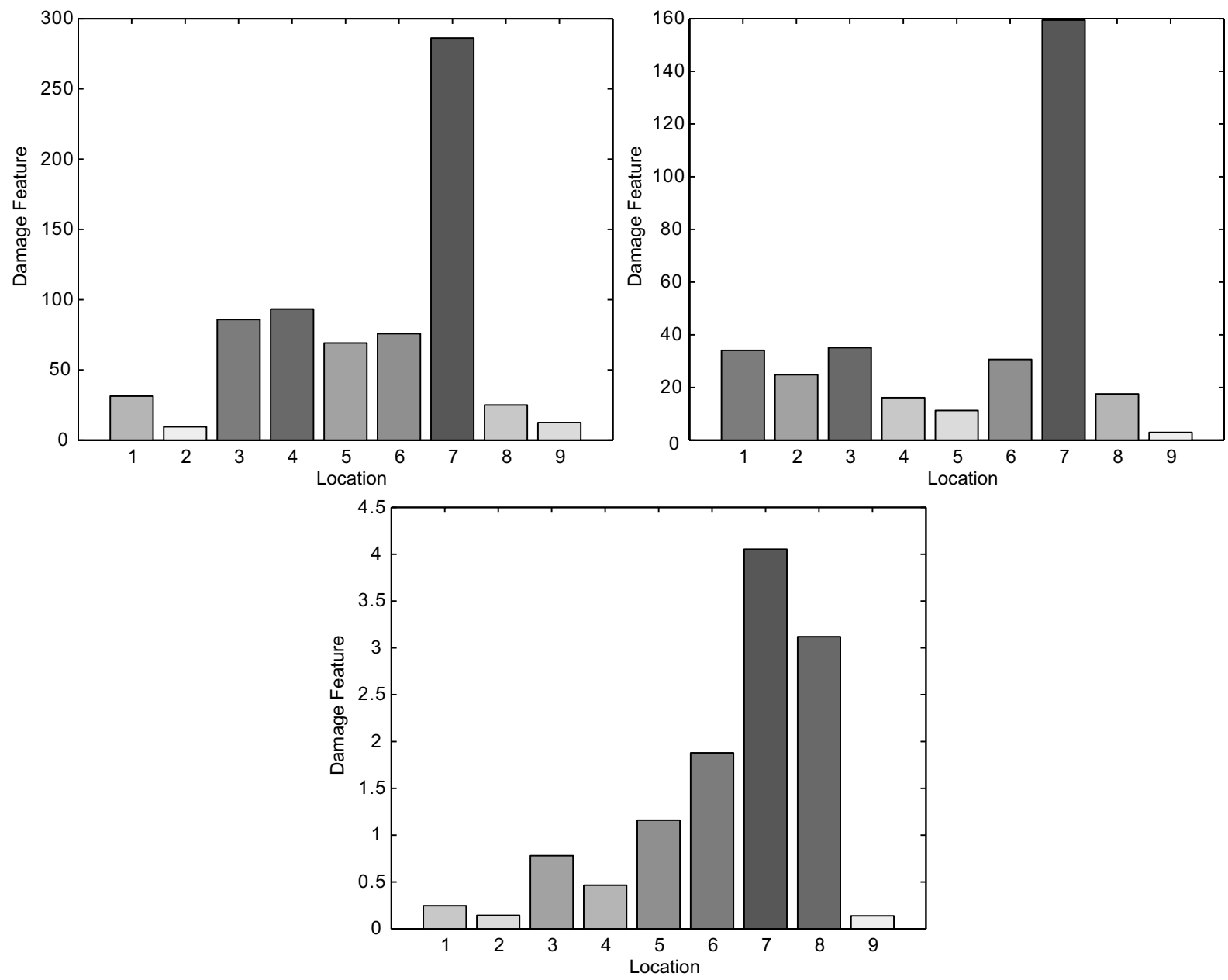

Fig. 6. Damage feature, frequency range 1-200 Hz (top left), frequency range $60-120 \mathrm{~Hz}$ (top right) and frequency range $10-40 \mathrm{~Hz}$.

the damage location. This can be explained due to the fact that not only the transmissibility function between the pair of responses where the damage is located is sensitive to the damage but also the other transmissibility functions are sensitive to the presence of the damage. This could clearly be seen in Fig. 5.

A possible approach to improve the results and to deal with this sensitivity of the transmissibility functions to non-local damage is a method based on occurrences. Instead of summing up the absolute difference between the transmissibility functions of the healthy and damaged structure at each frequency we can count an occurrence at each frequency at that location where the difference between the transmissibility function of the damaged and undamaged structure is a maximum. This means that if we have $N$ frequency lines in our frequency band of interest the total number of possible occurrences is $N$. The location which counts the most occurrences will also indicate the damage location.

In Fig. 7 one can see the damage feature using the approach of counting the occurrences for the frequency range $10-40 \mathrm{~Hz}$. It clearly shows that using occurrences improves in this frequency band the detection of the damage and therefore it was chosen in the rest of this paper to present always both approaches of the damage feature, without and with occurrences.

In the next paragraphs we will consider only those situations where the location of the operational force is changing before and after damage occurred. It will be demonstrated how the damage indicators might fail to locate the damage in these cases. First we have a look to the case when no damage occurs and how the change in transmissibility functions due to the change in loading conditions might result in false damage notifications. 


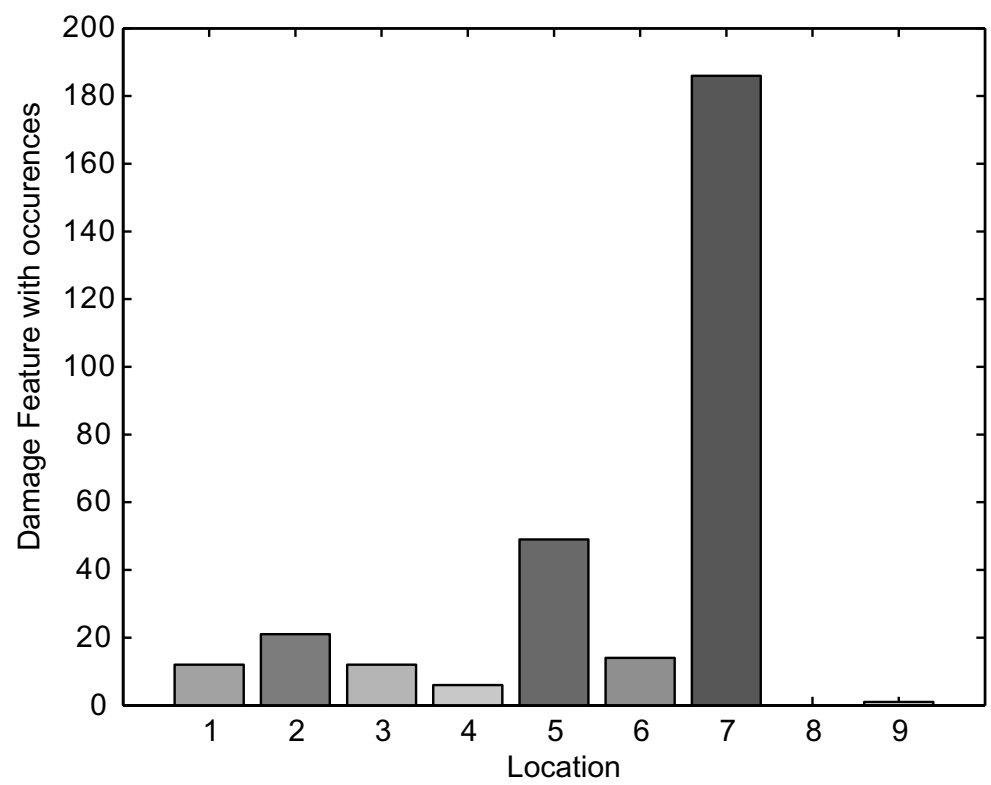

Fig. 7. Damage feature with occurrences, frequency range $10-40 \mathrm{~Hz}$.
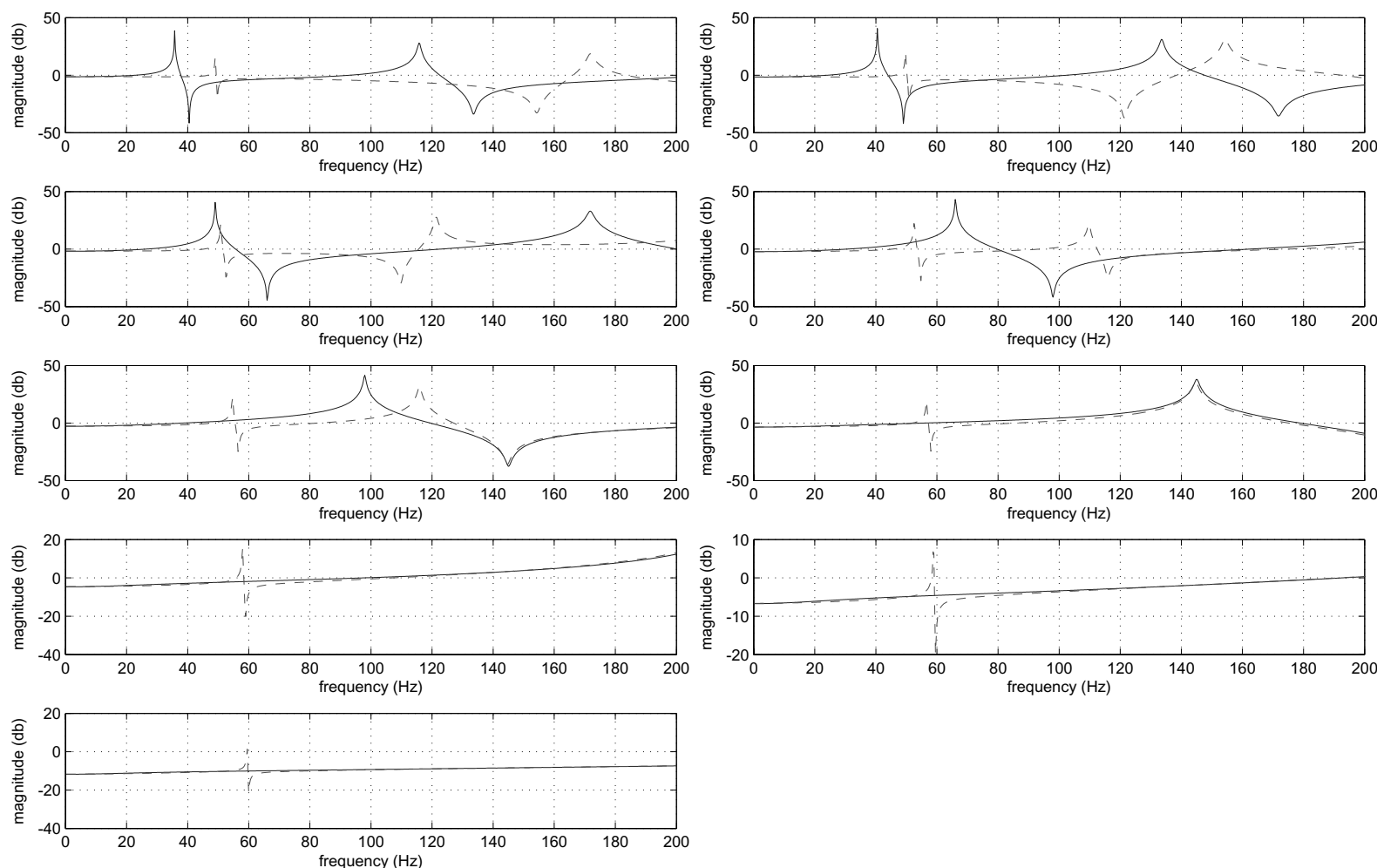

Fig. 8. Transmissibility functions measured on undamaged structure for loading case 1 (full line, force in DOF 1 ) and loading case 2 (dashed line, force in DOF 2), between responses at DOF 1 and DOF 2 (top left), DOF 2 and DOF 3 (top right) ... DOF 9 and DOF 10 (bottom left) 


\section{Problems of current damage indicators}

As mentioned in the introduction the problem rises when the location of the operational force changes during operation. Transmissibility functions as defined in Eq. (1) do depend on the location of the applied force.

$$
T_{i j(k)} \neq T_{j i(l)}
$$

Therefore a change in the location of the applied force would imply a noticeable change in the transmissibility functions. Using the above defined damage indicators for comparing transmissibility functions under different loading conditions would result in many non-zero values of the indicators at locations different from the damage location and inadvertently cause the mentioned techniques to fail. It is not unthinkable that these non-zero values mask the true location of the damage and make it impossible to perform a reliable structural health monitoring and false damage detection might occur in such situations, even in the case no damage is present.

\subsection{No damage is present and loading conditions change}

Figure 8 compares the transmissibility functions measured on the undamaged structure for the case the structure is excited in DOF 1 with those when the structure is excited in DOF 3 . One can clearly observe that all transmissibility functions changed due to the change of the location of the operational force.

These changes in the transmissibility functions have significant implications because they inadvertently cause the mentioned techniques to fail. In this case the damage feature, calculated by combining transmissibility functions of the healthy structure but under different loading conditions, gives several non - zero values at different locations. (Figs 9 to 11 ).

It can easily be understood that an undamaged structure will be falsely diagnosed with damage if the transmissibility functions are taken under varying loading conditions. Different frequency bands result also in different values of the damage feature and thus different false locations of damage.

\subsection{Damage is present and loading conditions change}

We already saw in the previous paragraph that false damage notifications may happen due to the change of the operational forces when the structure is healthy and no damage occurred. Even when the structure is damaged it might however become impossible to determine the correct location of the damage, if one arbitrarily chooses a frequency band. Often it may occur that the changes in the transmissibility functions due to the changing loading conditions are higher then the changes due to the damage. Therefore the high values of the damage feature at other locations can mask the true damage location. This can be observed in Figs 12 to 14.

In the next paragraph it will be shown how a proper selection of the frequency band can make the damage feature more reliable in order to correctly identify the location of the damage.

\section{Proposed solution in case of changing loading conditions}

One of the most interesting properties of transmissibility functions, recently shown in [7] is that the limit value of the transmissibility function Eq. (1) for $s$ going to the system's poles, $\lambda_{m}$, converges to

$$
\lim _{s \rightarrow \lambda_{m}} T_{i j(k)}(s)=\frac{\phi_{i m}}{\phi_{j m}}
$$

with $\phi_{i m}$ and $\phi_{j m}$ the scalar mode-shape values, and becomes independent of the (unknown) forces. This property does not depend on the location of the applied force

$$
\lim _{s \rightarrow \lambda_{m}} T_{i j(l)}(s)=\frac{\phi_{i m}}{\phi_{j m}}
$$

Therefore if we consider two transmissibility functions relating the same responses, but measured during two experiments with different loading conditions, they cross each other exactly at the resonance frequencies, where they 

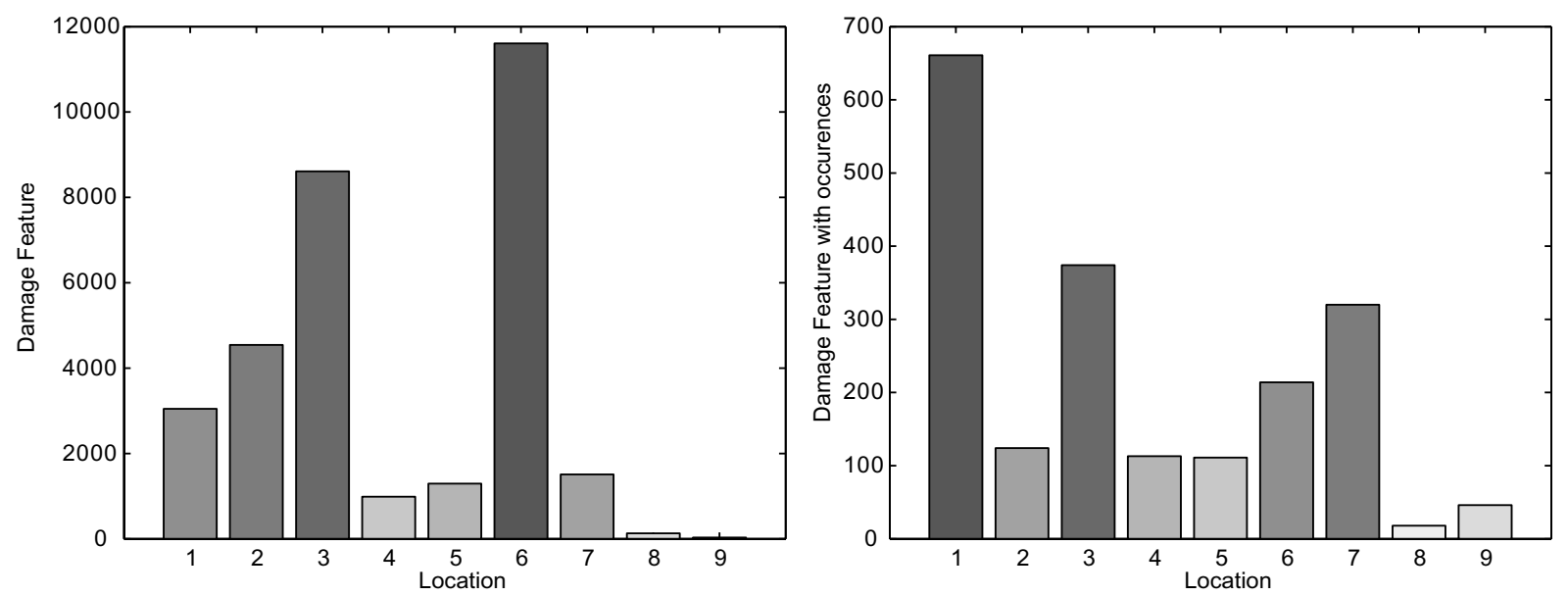

Fig. 9. Damage feature without and with occurrences for undamaged structure and changing loading conditions, frequency range 1-200 Hz.
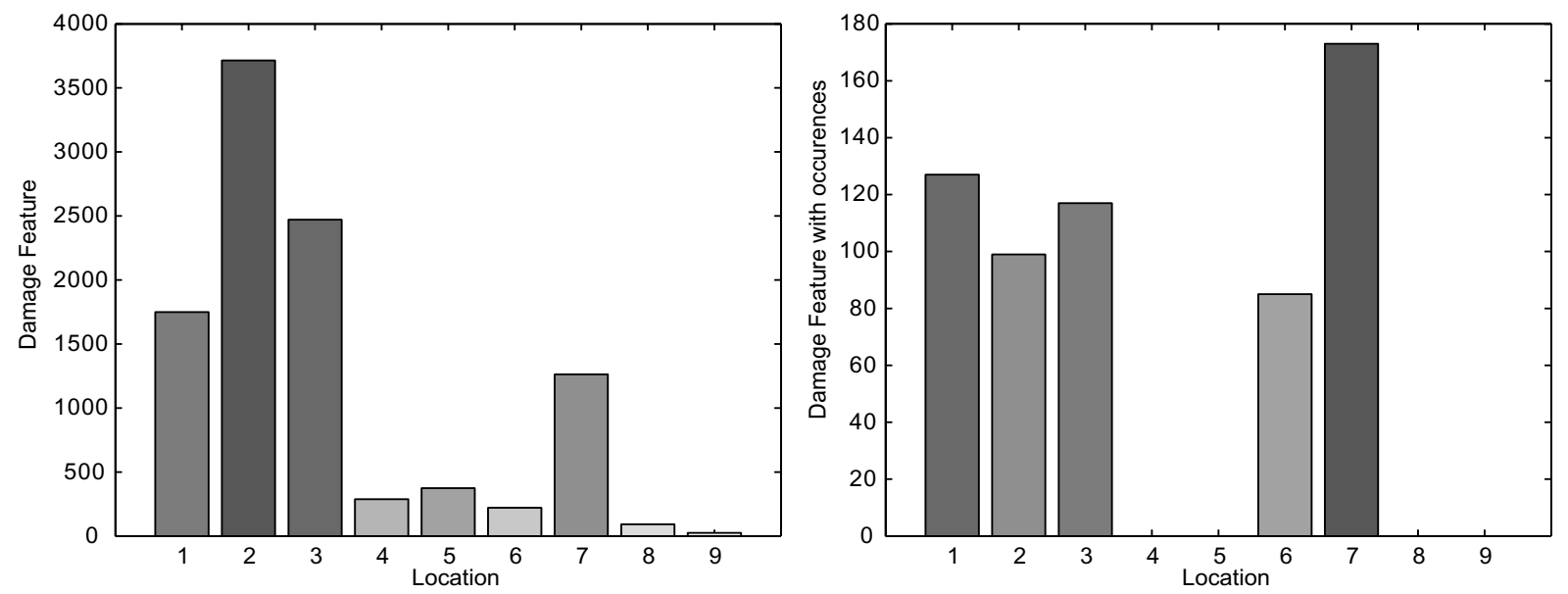

Fig. 10. Damage feature without and with occurrences for undamaged structure and changing loading conditions, frequency range $60-120$ Hz.
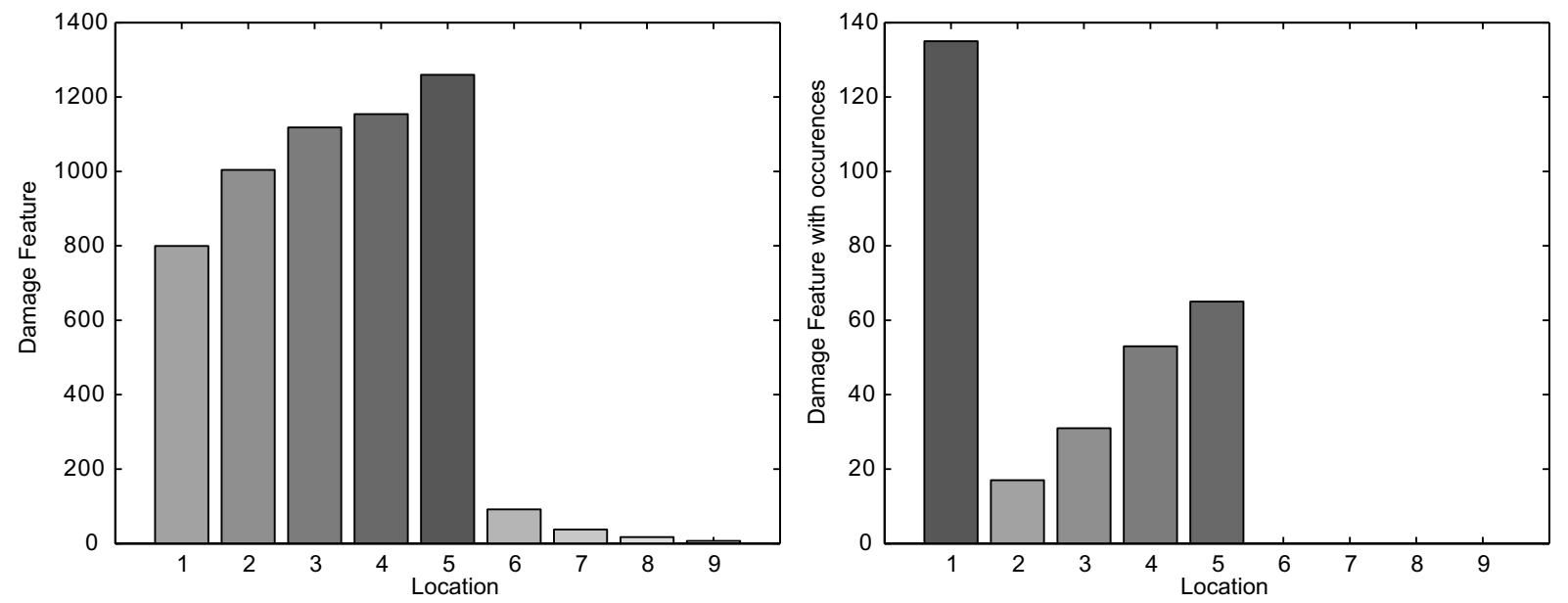

Fig. 11. Damage feature without and with occurrences for undamaged structure and changing loading conditions, frequency range $10-40 \mathrm{~Hz}$. 

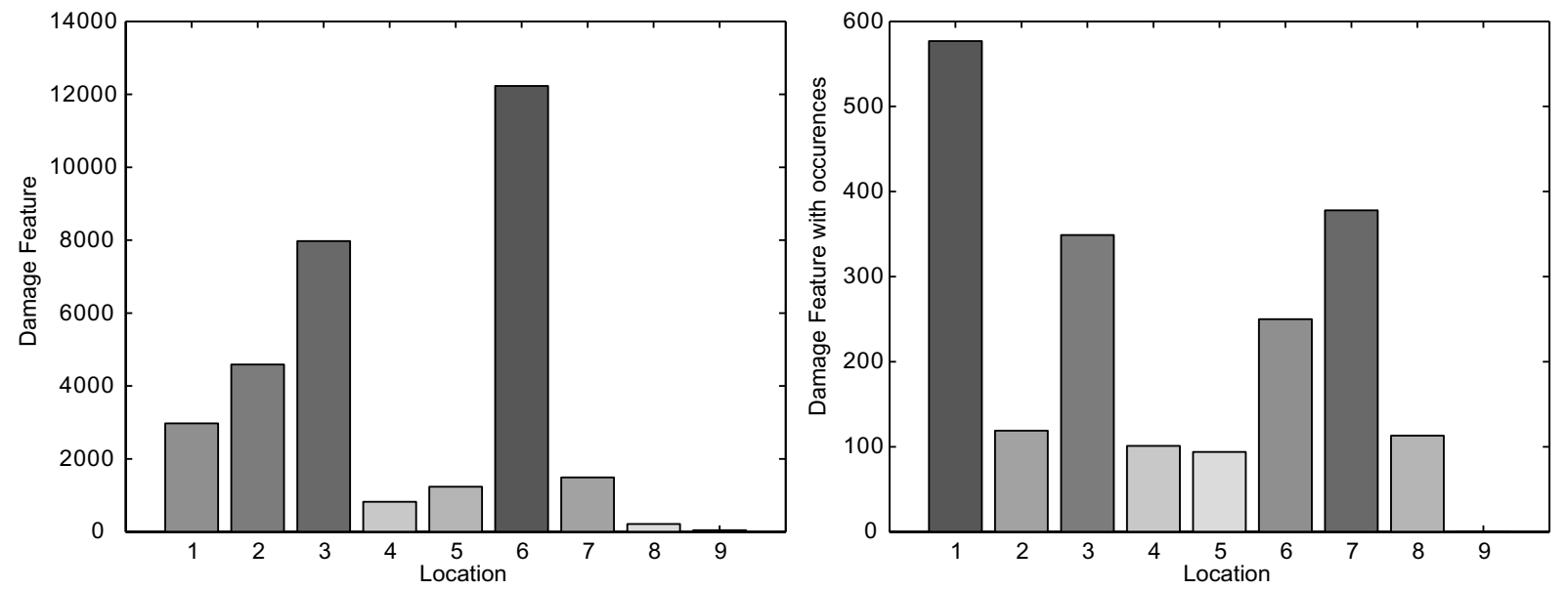

Fig. 12. Damage feature without and with occurrences for damaged structure and changing loading conditions, frequency range 1-200 Hz.
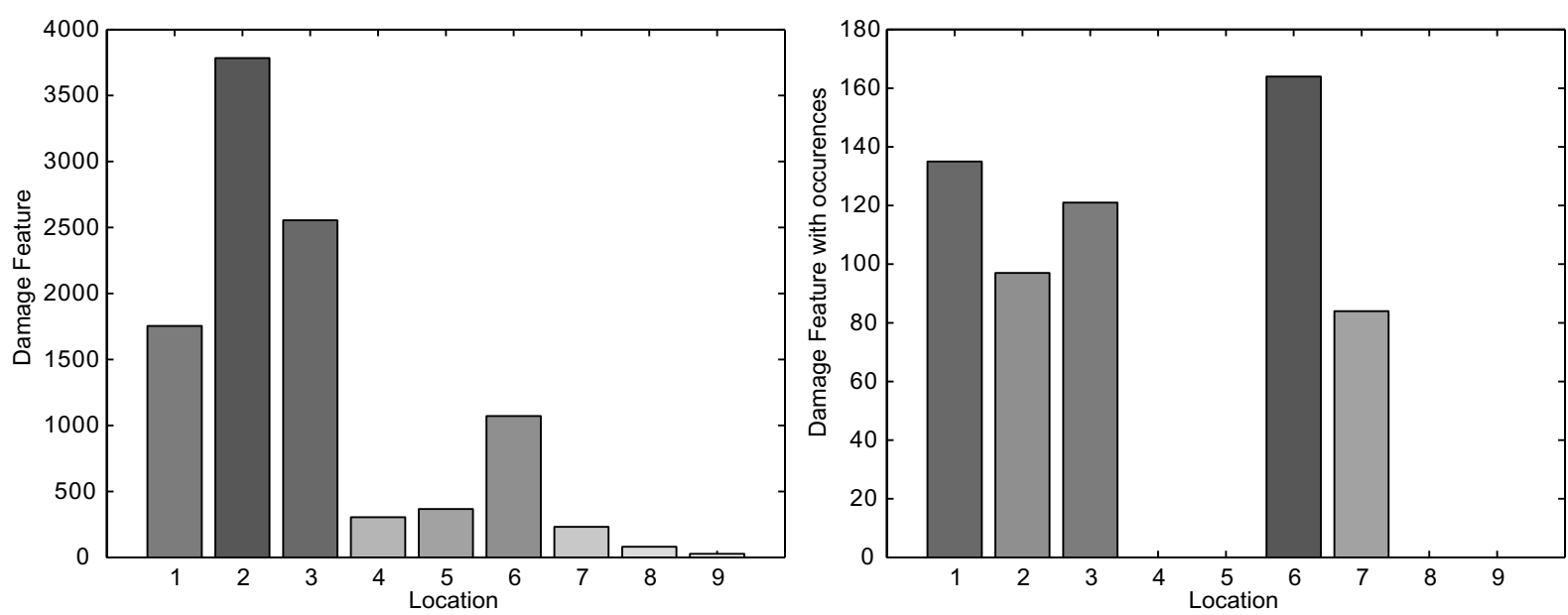

Fig. 13. Damage feature without and with occurrences for damaged structure and changing loading conditions, frequency range 60-120 Hz.
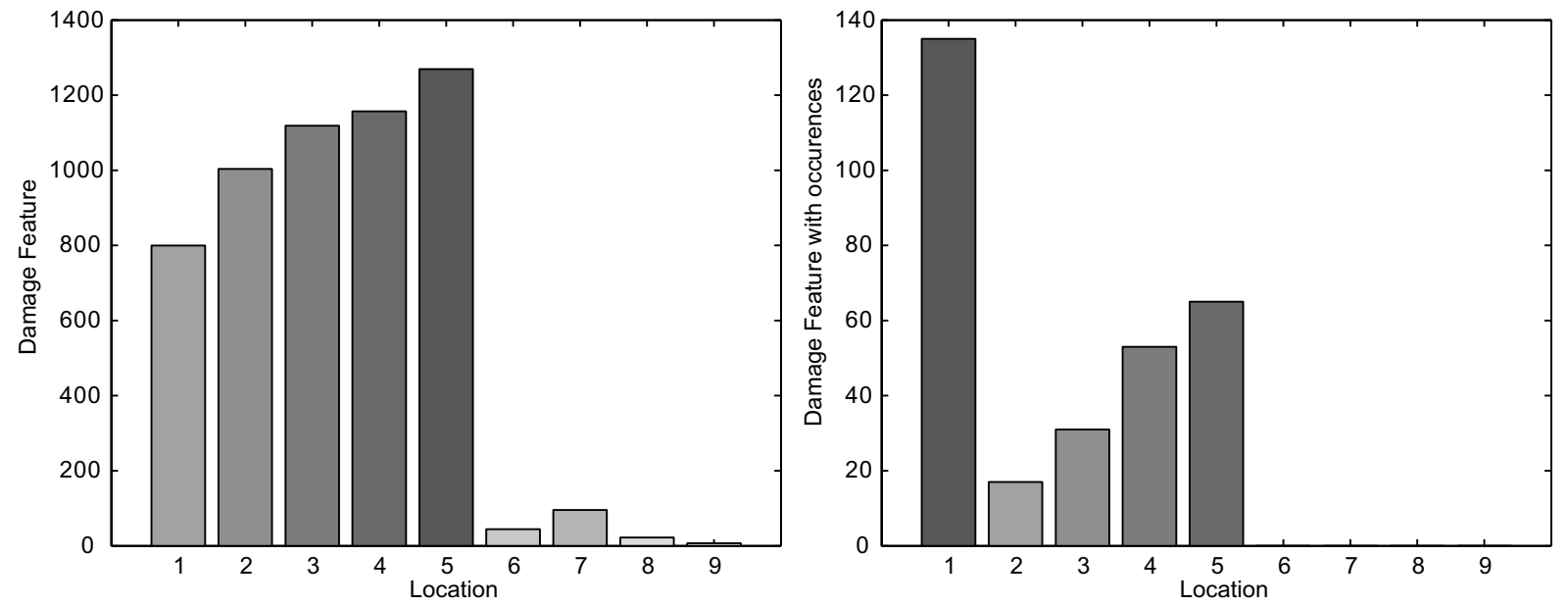

Fig. 14. Damage feature without and with occurrences for damaged structure and changing loading conditions, frequency range $10-40 \mathrm{~Hz}$. 


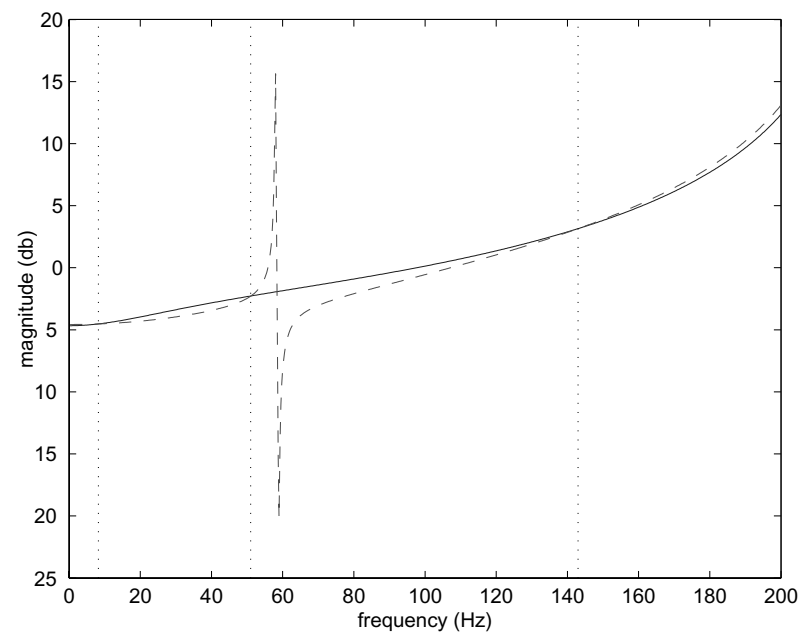

Fig. 15. Transmissibility function between point 7 and point 8 for undamaged structure for loading case 1 (full line, force applied in DOF 1 ) and loading case 2 (dashed line, force applied in DOF 3).
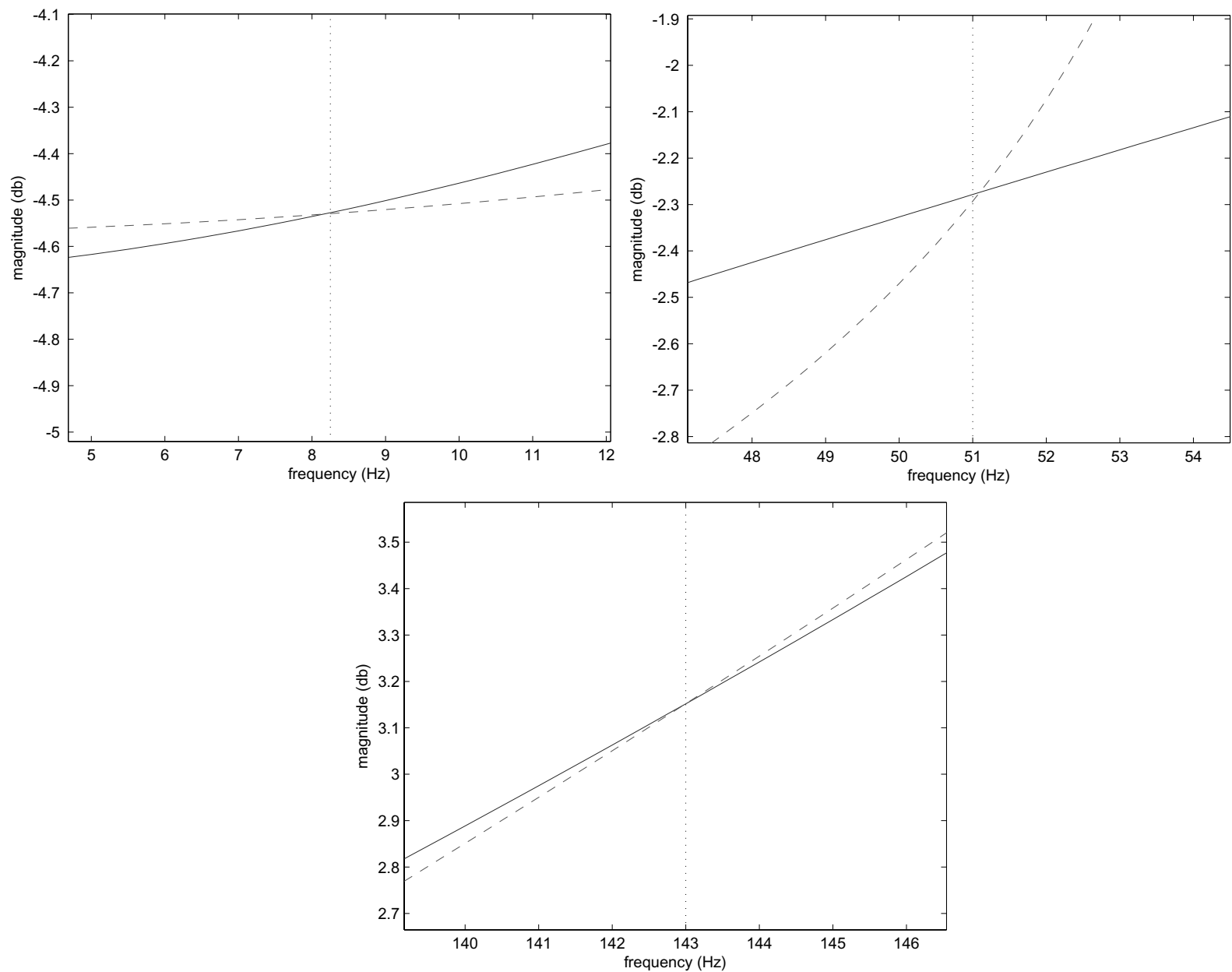

Fig. 16. Transmissibility function between point 7 and point 8 for undamaged structure for loading case 1 (full line; force applied in DOF 1 ) and loading case 2 (dashed line; force applied in DOF 1). 


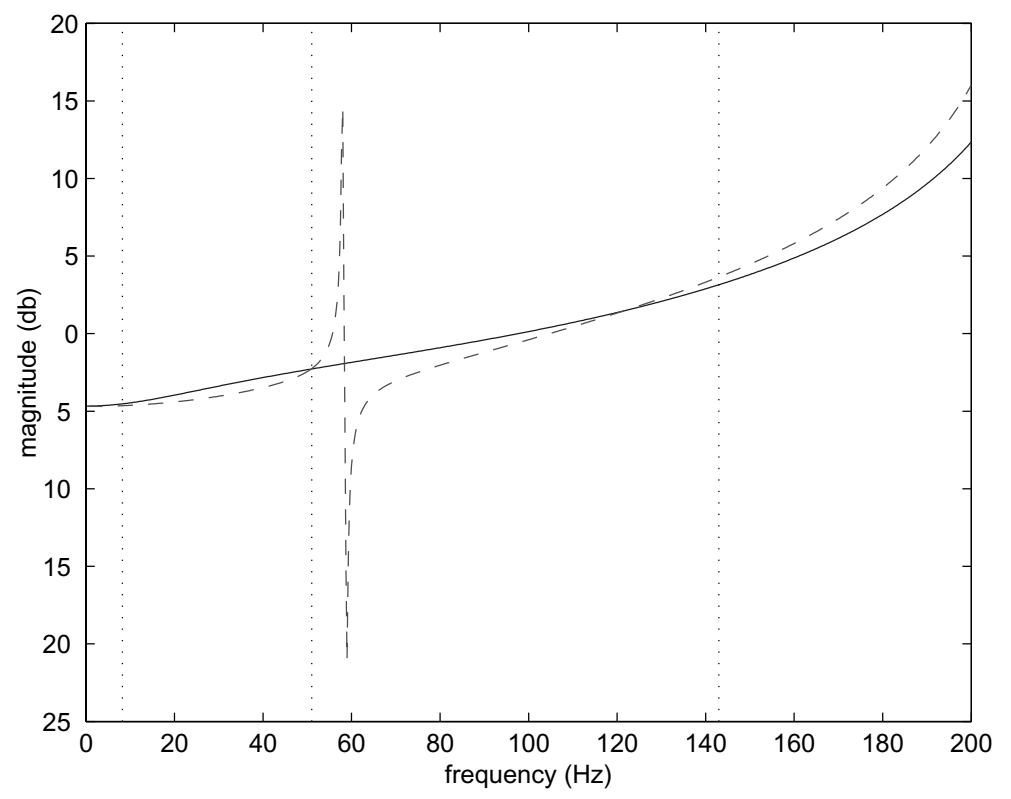

Fig. 17. Transmissibility function between point 7 and point 8 for undamaged structure for loading case 1 (full line; force applied in DOF 1 ) and damaged structure for loading case 2 (dashed line, force applied in DOF 3).

become independent of the loading condition. This property can clearly be seen in Fig. 15, where the transmissibility function between point 7 and point 8 during the two different loading conditions is shown. The vertical dashed lines are located at the resonance frequencies of the structure. A zoom around the resonance frequencies can be seen in Fig. 16.

This knowledge can already be used as a simple indicator to eliminate false damage notifications. One could measure transmissibility functions of the structure over a successive time under varying loading conditions. Only when the intersection points of the transmissibility functions under varying loading conditions start to shift, due to the shift of the natural frequencies of the structure, damage is occurring. In Fig. 17 the full line represents the transmissibility function between point 7 and point 8 for the undamaged structure and the dashed line is the same transmissibility function after damage occurred and for a different loading condition. One can clearly observe that the transmissibility functions still cross but no longer at the resonance frequencies of the healthy structure.

By looking to a zoom around the resonance frequencies in Fig. 18 one can give the same conclusions as before with the responses in Fig. 3. The damage, characterized by the shift of the crossing point between 2 transmissibility functions, is most obvious around the first and third resonance frequency. We know already that the second mode is almost not influenced by the damage.

This brings us to the essence of the proposed approach in this paper. In order to locate the damage, using the existing damage indicators, in the case the location of the applied force on the healthy structure is different than the location of the applied force on the damaged structure, a careful choice of the selected frequency band is proposed. Taking into account that the transmissibility functions at the resonance frequencies do not depend on the loading conditions, as is shown in Eqs (9) and (10), the proposed choice would be to select only a small frequency band around these resonance frequencies in order to calculate the damage feature. Assuming that in this frequency band the change in the transmissibility functions is mainly due to the presence of damage then due to the changing loading conditions.

We will now have a look to the proposed technique. Therefore we will select only a small frequency band around the resonance frequencies of the structure in order to calculate the damage feature. The next figures demonstrate this approach (Figs 19 and 20). It is in line with the expectations that for the damage feature calculated in a small frequency band, 7-9 Hz around the first resonance frequency (Fig. 19) and 142-144 Hz around the third resonance frequency (Fig. 20) the damage is correctly located at location 7. In both cases the approach using occurrences results in fewer non-zero values at locations without damage. 

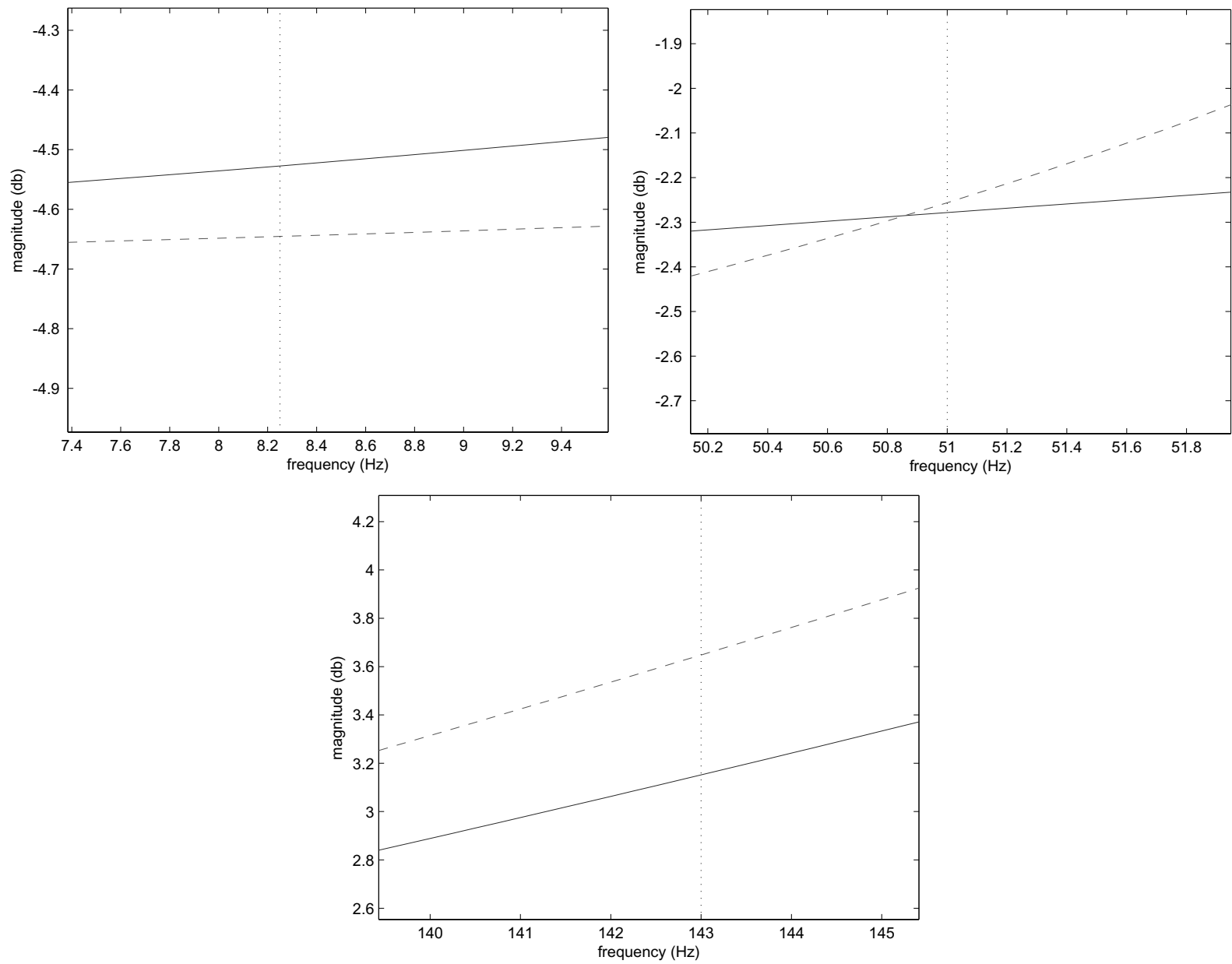

Fig. 18. Transmissibility function between point 7 and point 8 for undamaged structure for loading case 1 (full line, force applied in DOF 1 ) and damaged structure for loading case 2 (dashed line, force applied in DOF 3).
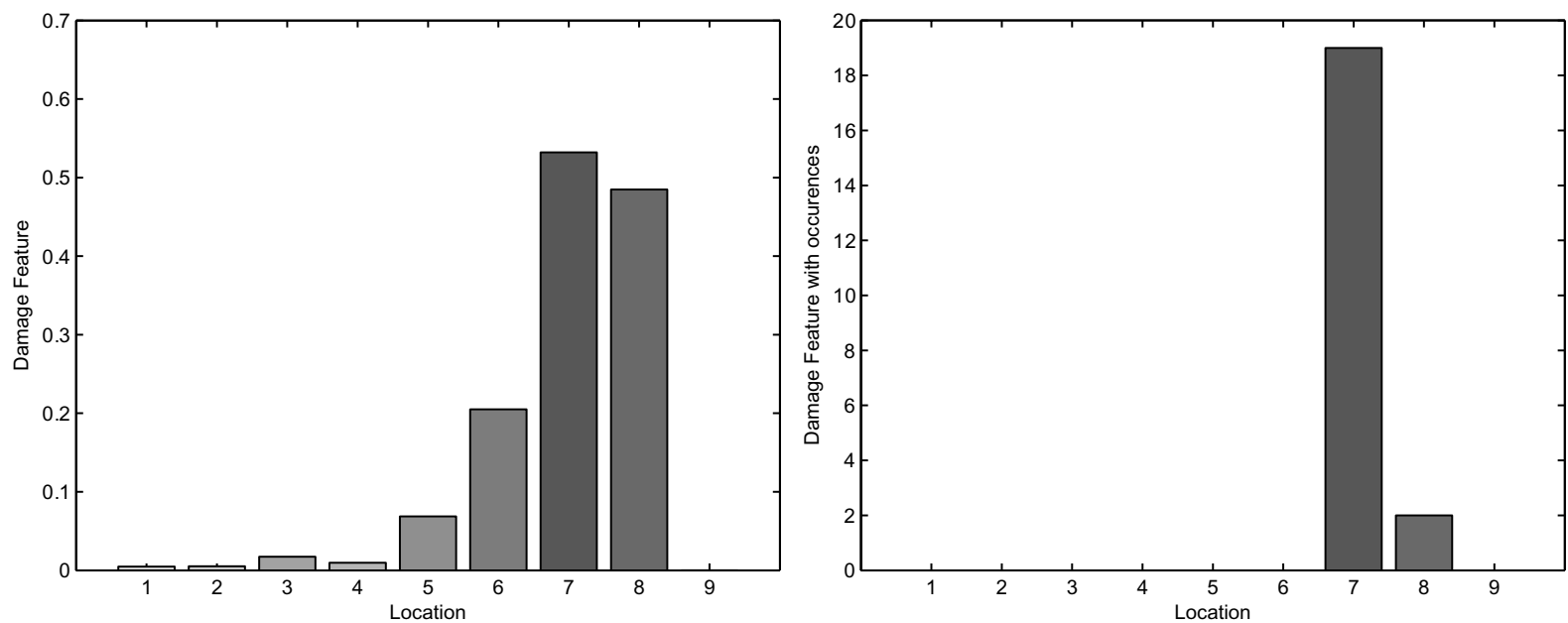

Fig. 19. Damage feature without and with occurrences for damaged structure and changing loading conditions, frequency range $7-9$ Hz. 

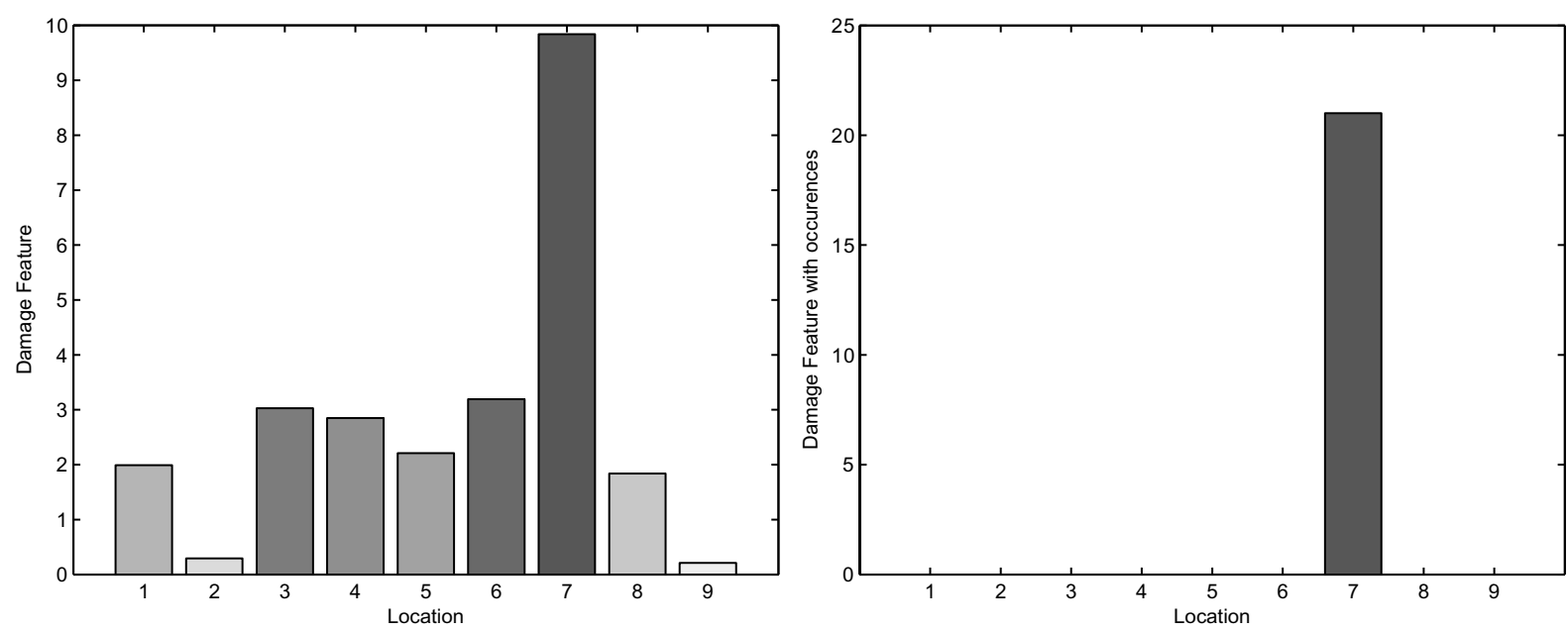

Fig. 20. Damage feature without and with occurrences for damaged structure and changing loading conditions, frequency range 142-144 Hz.
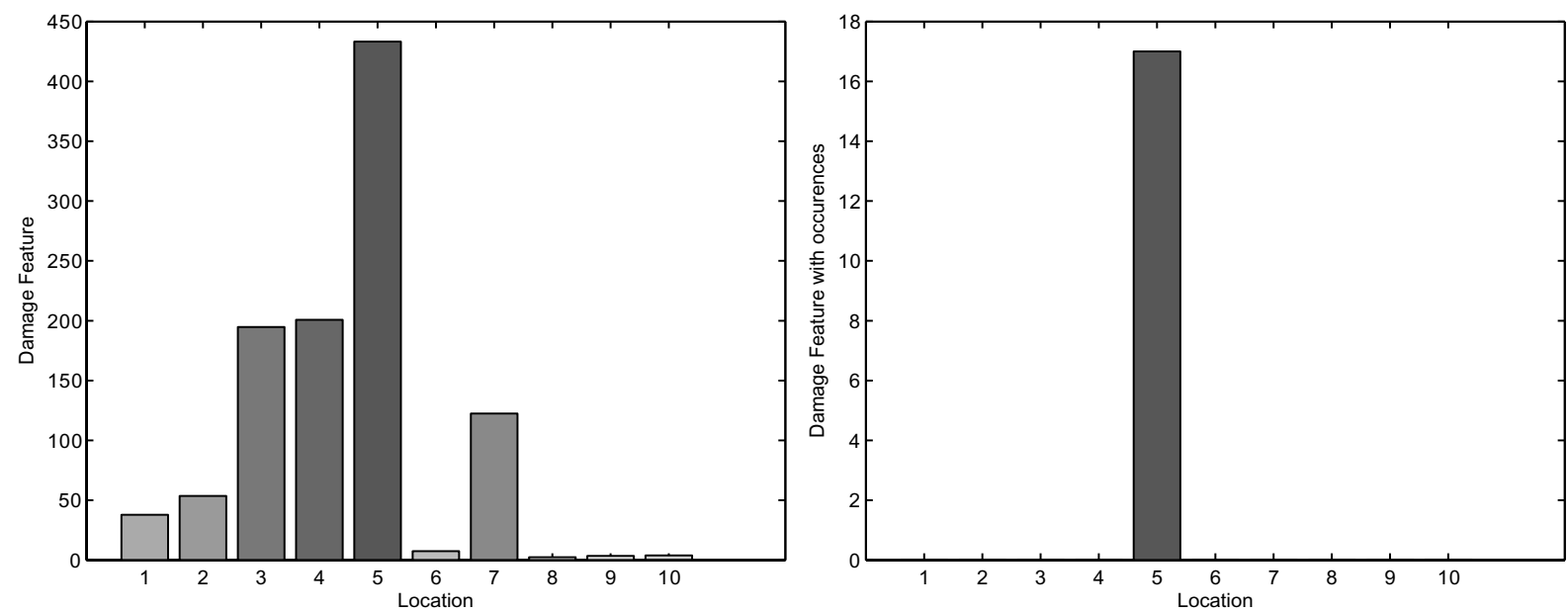

Fig. 21. Damage feature without and with occurrences for damaged structure and changing loading conditions, frequency range $50.5-51.5 \mathrm{~Hz}$.

Around the second resonance frequency the identification of the damage location is not possible (Fig. 21). This could be expected considering the fact that the second mode was almost not affected by the damage and thus the frequency band around the second resonance frequency contains few valuable information about the damage. One can conclude that the damage feature in case of changing loading conditions is only reliable in detecting damage around a sufficient small band around the resonance frequencies of those modes that were affected by damage.

\section{Remarks about proposed solution}

In the next two paragraphs we have a look to what happens if we take larger frequency bands around the resonance frequencies or small frequency bands next to the resonace frequencies. It is expected that in these bands the influence of the varying loading conditions will again be more important then the damage and will make reliable damage location impossible. 

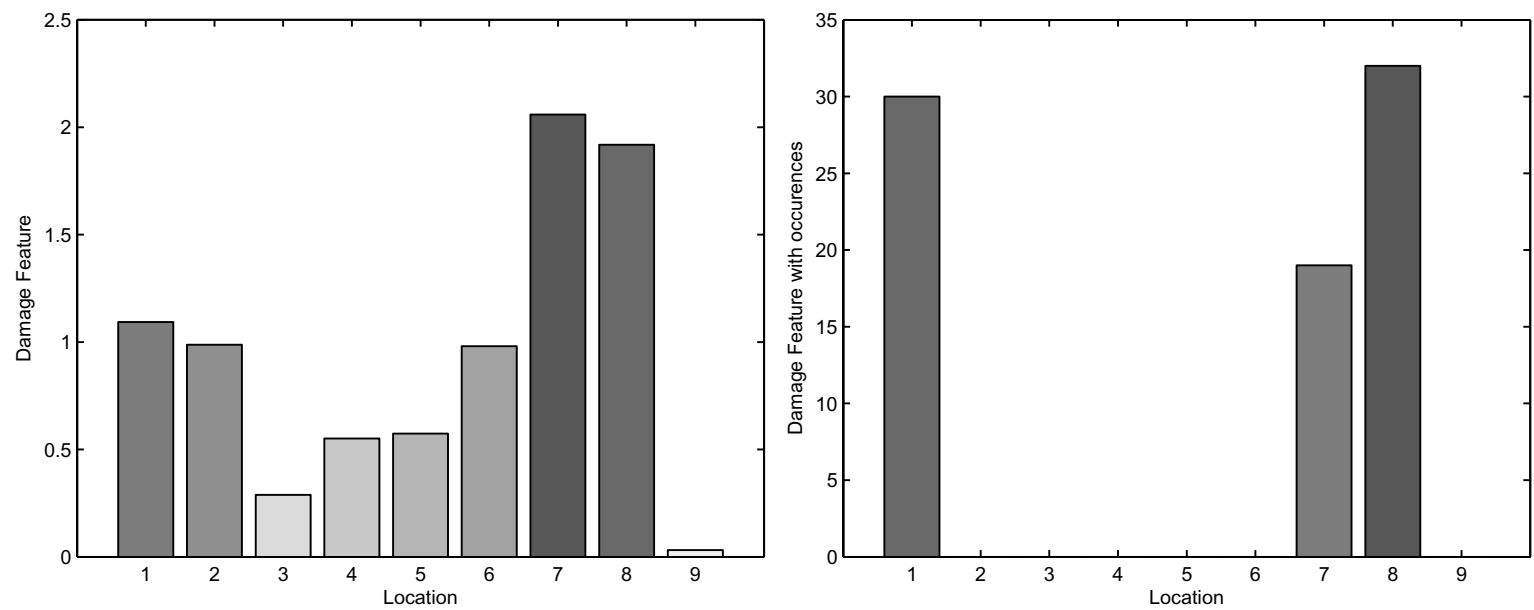

Fig. 22. Damage feature without and with occurrences for damaged structure and changing loading conditions, frequency range 4-12 Hz.
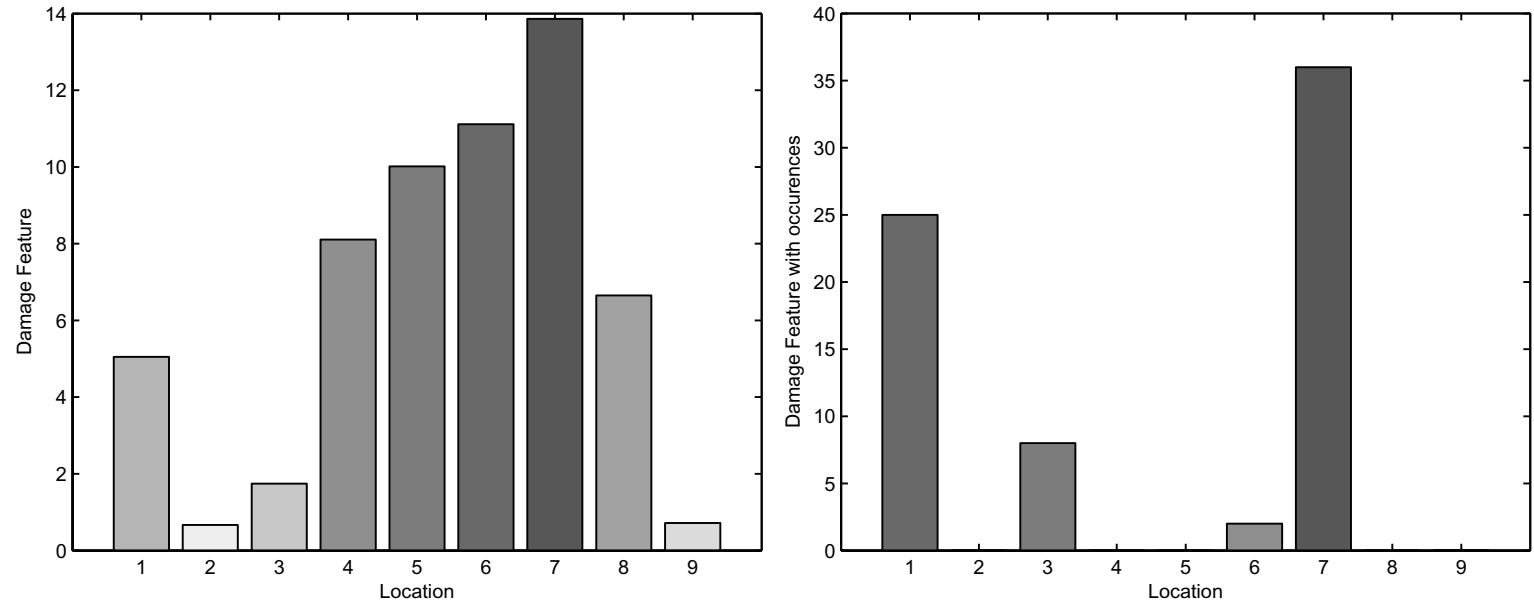

Fig. 23. Damage feature without and with occurrences for damaged structure and changing loading conditions, frequency range 139-146 Hz.
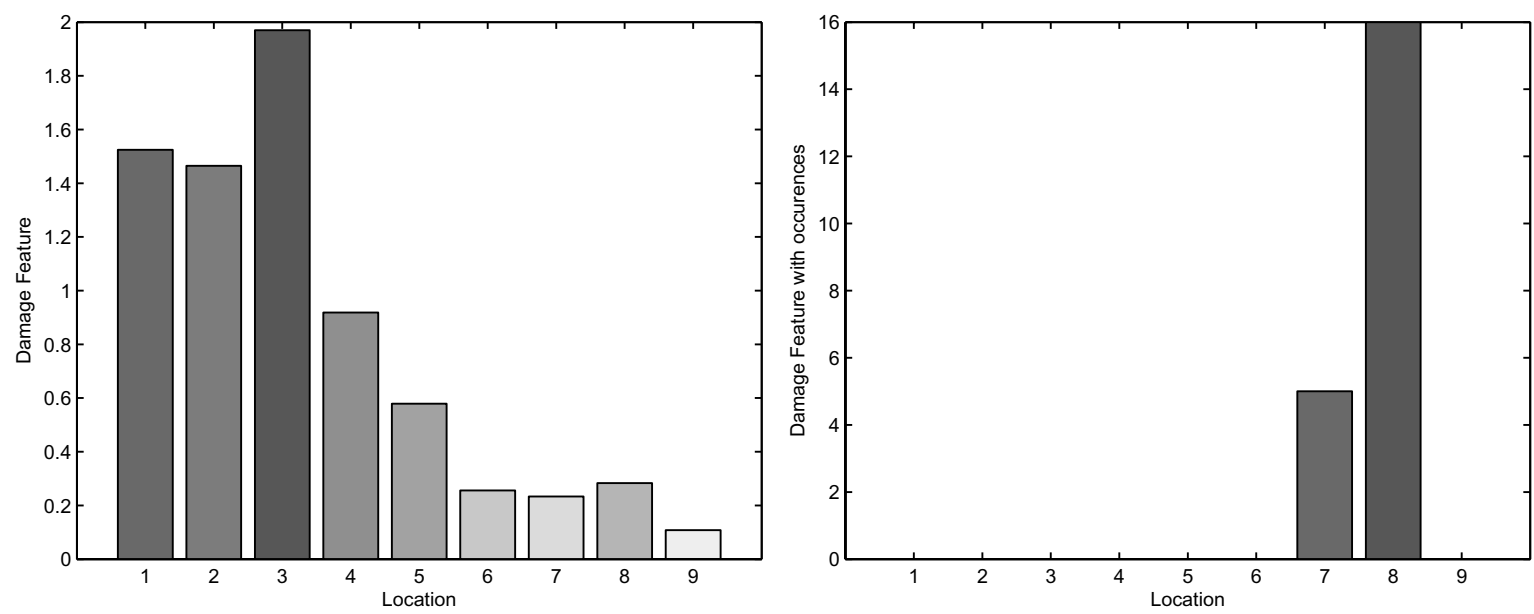

Fig. 24. Damage feature without and with occurrences for damaged structure and changing loading conditions, frequency range 3-5Hz. 

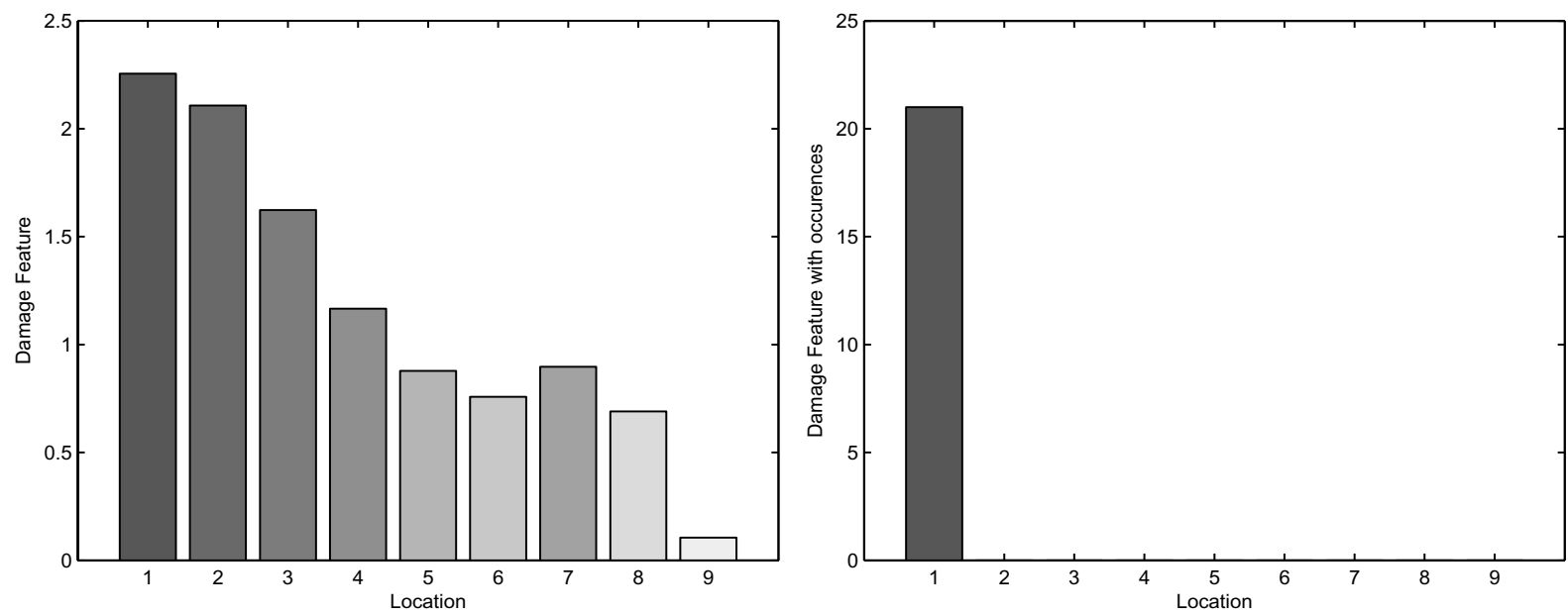

Fig. 25. Damage feature without and with occurrences for damaged structure and changing loading conditions, frequency range 10-12Hz.

\subsection{Using larger frequency bands around the resonance frequencies}

In Figs 22 and 23 one can clearly see that by taking a larger frequency band around the resonance frequencies the non-zero values of the damage feature at other location than where the damage occurred increases. This makes it harder to correctly identify the true location of the damage.

\subsection{Using small frequency bands not around the resonance frequencies}

In Figs 24 and 25 one can see that the damage identification fails when one takes a small band left or right from e.g. the first resonance frequency.

\section{Experimental validation}

To validate the use of transmissibility functions in changing operational loading conditions for structural health monitoring an experiment is performed using an aluminum cantilever beam $(0.7 \mathrm{~m} \times 0.03 \mathrm{~m} \times 0.005 \mathrm{~m})$. The operational force is provided by the use of pneumatic excitation. This excitation method was recently proposed in an article by Vanlanduit et al. [9] as an interesting alternative for non-fixed excitation methods in modal analysis (Fig. 26). During the measurements the full setup was placed on the ground to avoid possible influences due to the flexibility of the table.

During the experiments a pulse was used and the measurements where performed in a frequency range of 10 $100 \mathrm{~Hz}$. A total of 11 responses where measured by means of a Polytec Laser Vibrometer in equally spaced points along the length of the beam (Fig. 26). The frequency resolution is $0.125 \mathrm{~Hz}$ and no averages were performed. The numbering of the DOFs start from the free end of the beam. The structure was damaged by making a saw cut between DOF 7 and DOF 8 (Fig. 26). Two loading conditions were considered. During the first loading condition the air excited the structure in DOF 1 and during the second loading condition the beam was excited in DOF 4 . The two loading conditions were applied before and after damage occurred.

The measured responses, while the structure was excited in DOF 1, before (full line) and after damage (dashed line) was applied are shown in Fig. 27. In the frequency range of interest two resonance frequencies are present, respectively at $13.625 \mathrm{~Hz}$ and $85.125 \mathrm{~Hz}$ (for the undamaged case). A small change in the responses due to the presence of damage can be noticed. In Fig. 28 one can observe the shift in both the resonance frequencies due to the damage.

Figure 29 gives the transmissibility functions for the undamaged and damaged case for the two loading conditions. By trying to understand this figure some interesting conclusions, that are in line with the theory and the results from 

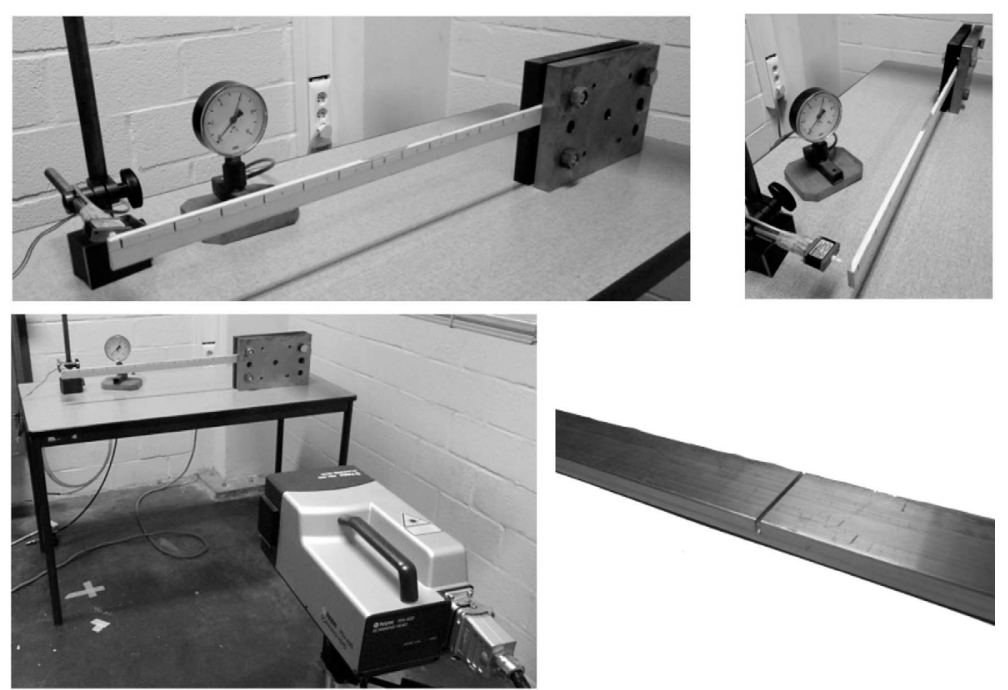

Fig. 26. Setup of the cantilever beam experiment with pneumatic excitation (top left), the use of the pneumatic excitation showing the valve and the nozzle (top right), measurement with laser Doppler vibrometer (bottom left), saw cut in aluminum beam (bottom right).

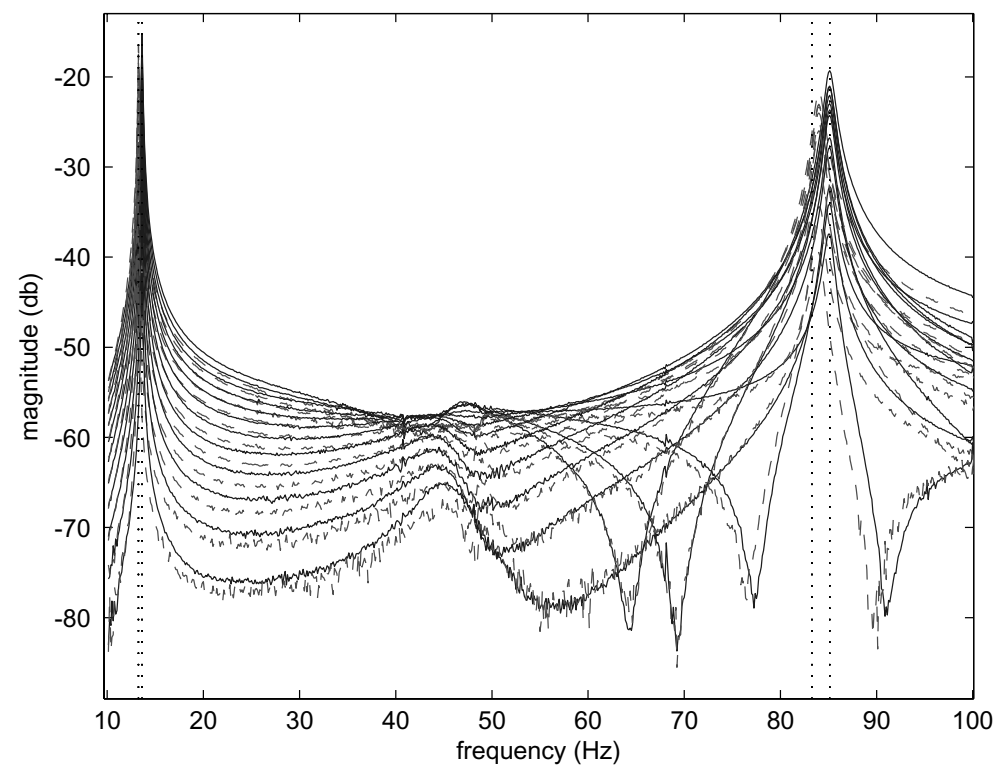

Fig. 27. Response functions for undamaged (full line) and damaged case (dashed line) with excitation in DOF 1 for both cases.

the above simulations, can be given. One can distinguish two zones of interest. The zones around the resonance frequencies and those away from the resonance frequencies. In the zone away from the resonance frequencies the transmissibility functions related with the same loading condition tend to coincide, while in the zone around the resonance frequencies the transmissibility functions related with the same health condition tend to converge to each-other.

We can conclude from this that in the zones around the resonance frequencies the difference between the different transmissibilities is mainly due to the difference in health condition and away from the resonance frequencies the differences between the transmissibility functions is mainly due to a change in loading conditions. Therefore the only zone where we might have a reliable damage indication in case of changing loading conditions is in a small frequency band around the resonance frequencies. 

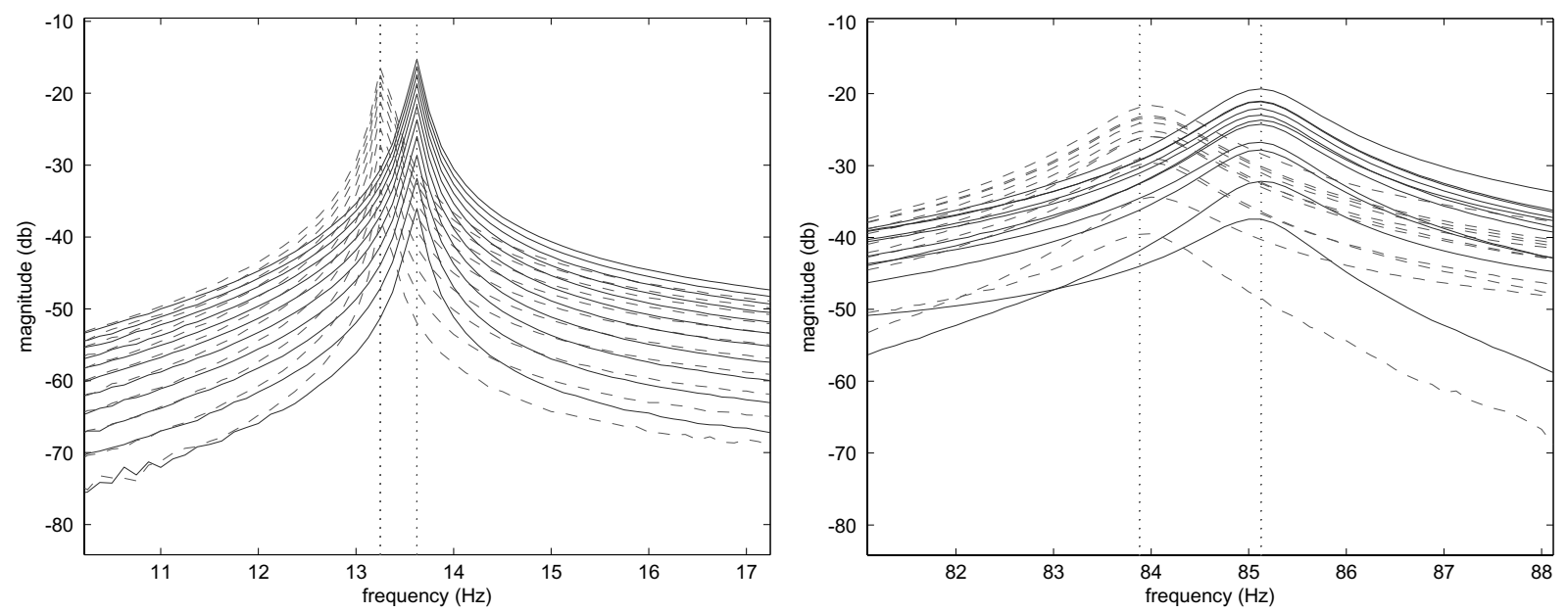

Fig. 28. Zoom on response functions for undamaged (full line) and damaged case (dashed line) with excitation in DOF 1 for both cases.

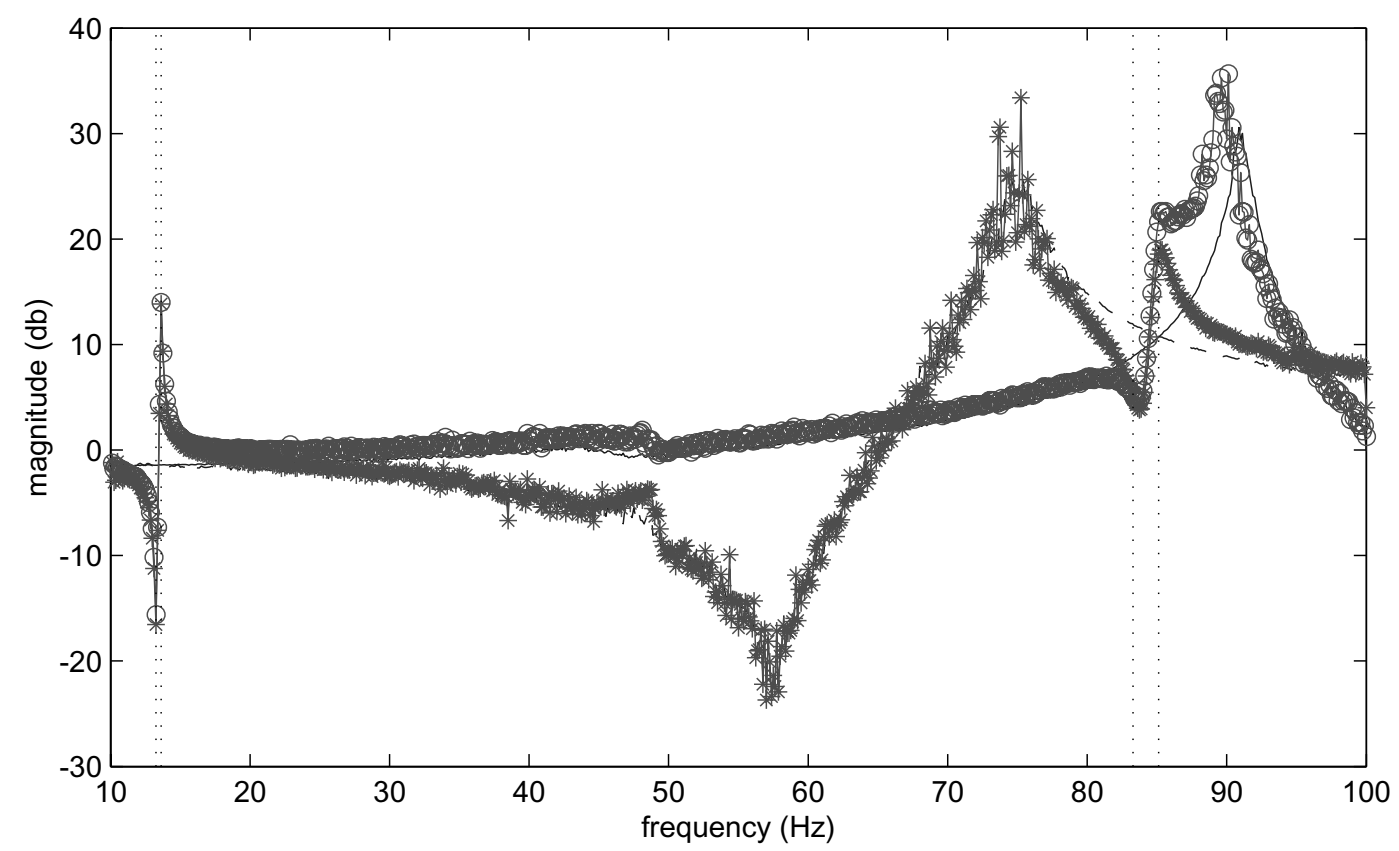

Fig. 29. Transmissibility functions for undamaged case and first loading condition (full line), undamaged case and second loading condition (dashed line), damaged case and first loading condition (full line with o) damaged case and second loading condition (full line with *).

In the next paragraphs we are going to analyze the performance of the damage feature without and with occurrences on the different scenarios of the experiment. First we have a look at the performance of the damage indicator in case of constant loading conditions. Secondly we will analyze the situation of changing loading conditions and the influence of the frequency band on the damage indicator.

\subsection{Constant location of operational force before and after damage}

\subsubsection{Using arbitrarily chosen frequency bands}

In this case the change in transmissibility function is only due to the damage and the corresponding change of the dynamic properties of the beam. The beam was only excited at DOF 1 so the loading conditions can be considered 

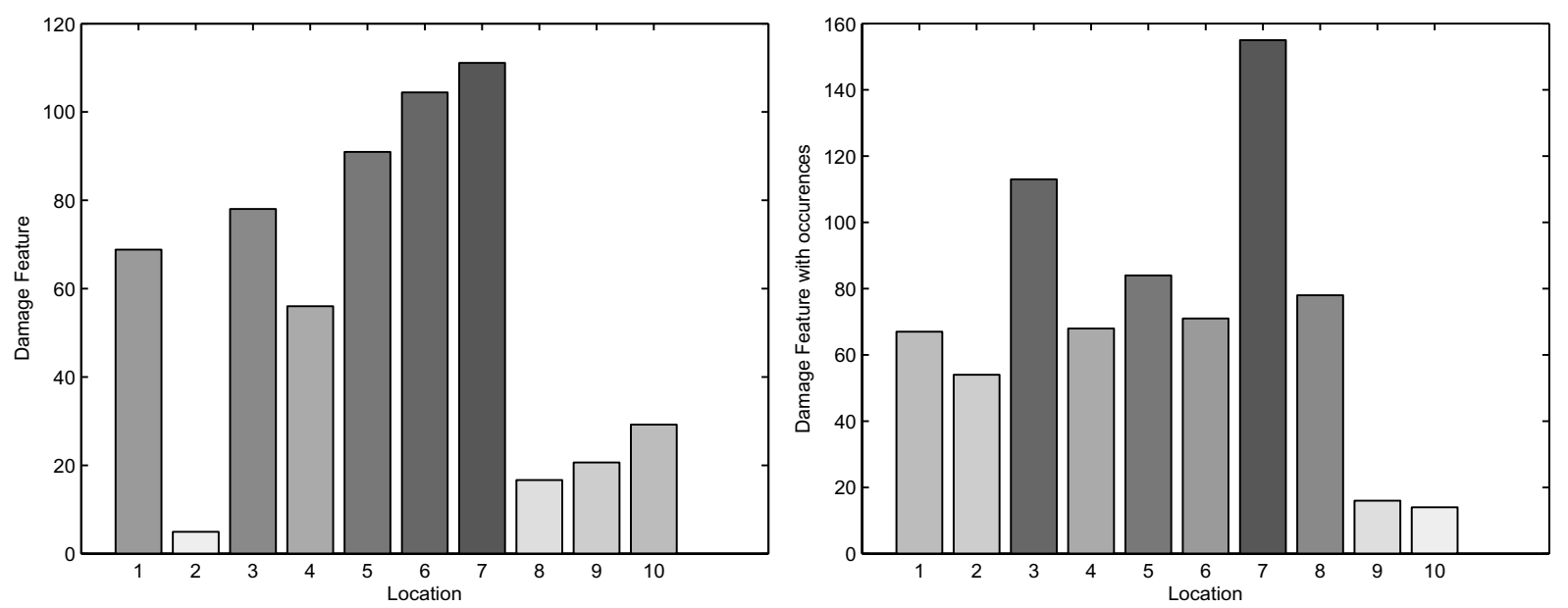

Fig. 30. Damage feature without and with occurrences for damaged structure and constant loading conditions (force applied in DOF 1), frequency range $10-100 \mathrm{~Hz}$.
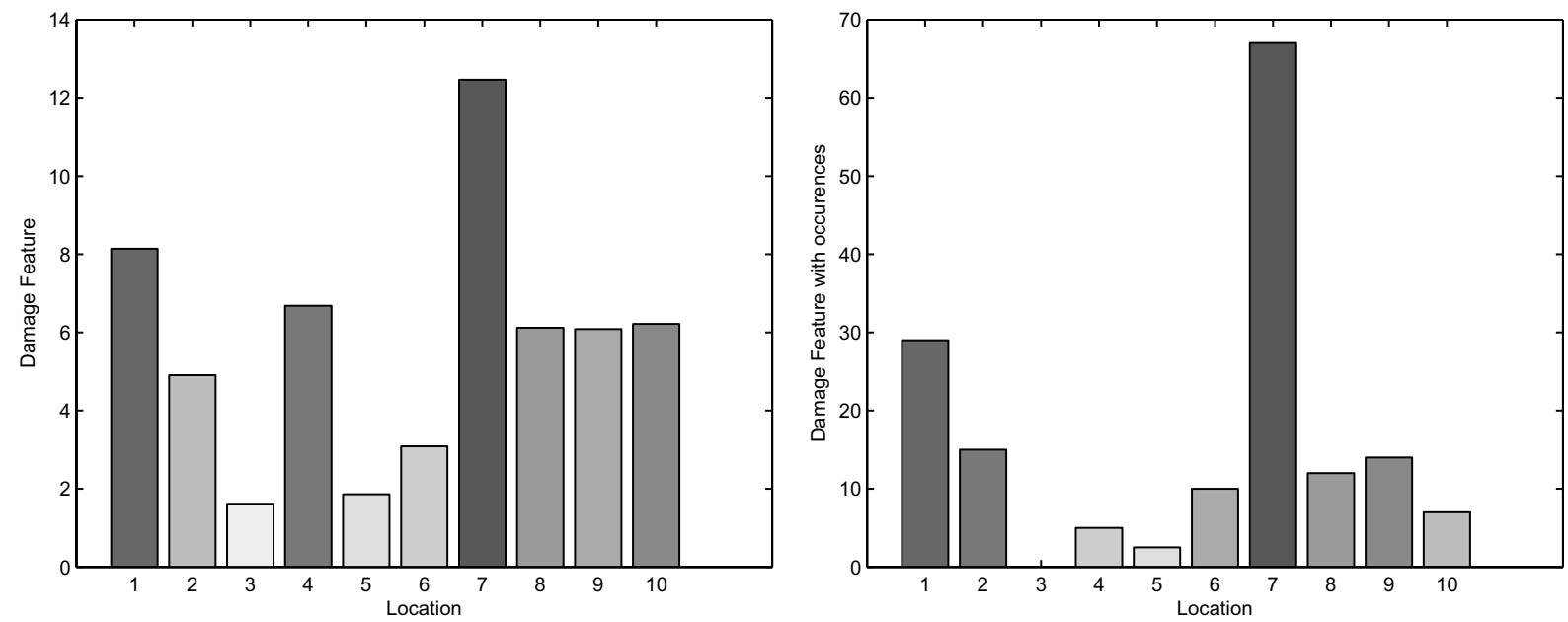

Fig. 31. Damage feature without and with occurrences for damaged structure and constant loading conditions (force applied in DOF 1), frequency range $10-30 \mathrm{~Hz}$.

constant before and after the damage occured. Two frequency bands were arbitrarily selected to calculate the damage indicator, namely the frequency band $10-100 \mathrm{~Hz}$ and the frequency band $10-30 \mathrm{~Hz}$. The damage feature was again calculated without and with using the occurrences approach (Figs 30 and 31). In all cases the highest values of the indicator is at location 7 , the correct location of the damage.

Several non-zero values of the damage feature are however present at other locations then the location of the damage making easy and reliable damage identification rather difficult. This can be explained partly due to the presence of the noise and the fact that the results tend to degenerate when we go along the frequency range and add more and more information masking the true location of the damage.

Using the occurrences approach seems to improve the results especially in the smaller frequency band of $10-30 \mathrm{~Hz}$ (Fig. 31). One can conclude that in practice in the case of constant loading conditions the use of small low frequency bands combined with the occurrences approach is often the most reliable solution. This conclusions was also given by Maia et al. [4]. 

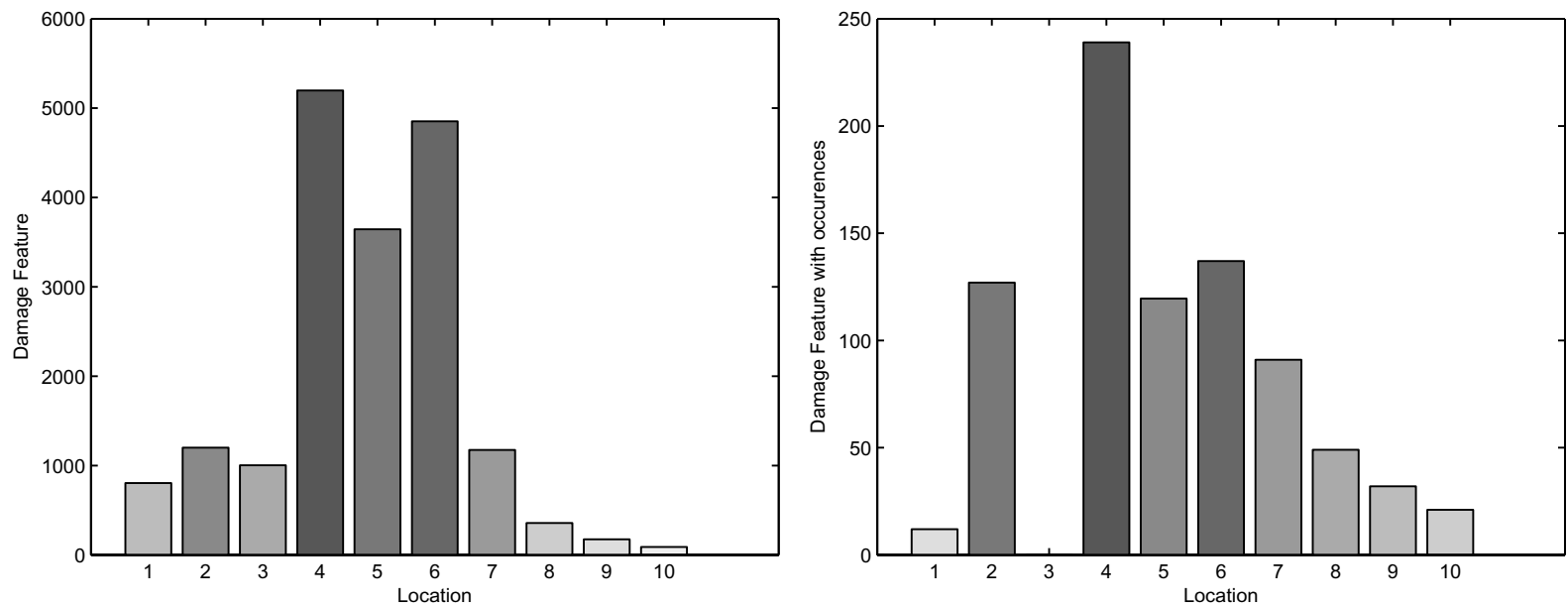

Fig. 32. Damage feature without and with occurrences for damaged structure and changing loading conditions, frequency range 10-100 Hz.
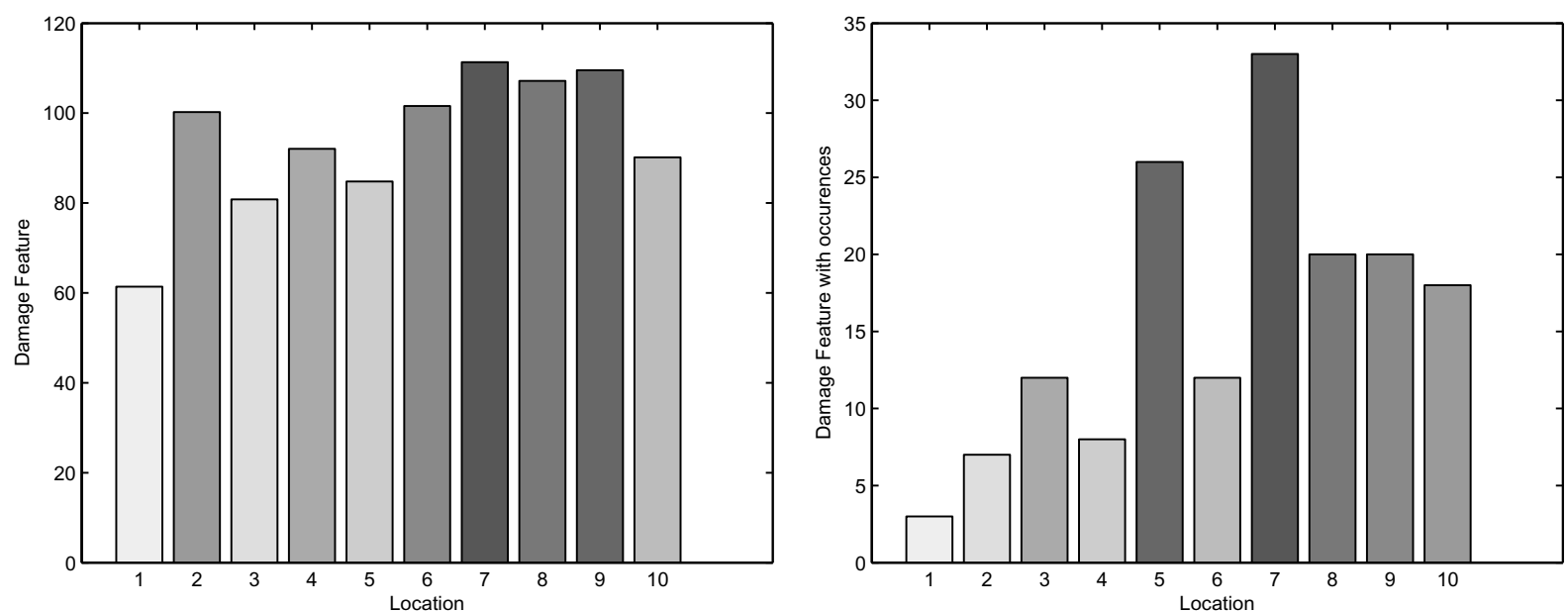

Fig. 33. Damage feature without and with occurrences for damaged structure and changing loading conditions, frequency range 10-30Hz.

\subsection{Different location of operational force before and after damage}

We now have a look to the case of changing operational conditions. In the first loading condition the beam was excited by the pressurized air in DOF 1 . In the second loading condition the beam was excited in DOF 4 .

\subsubsection{Using arbitrarily chosen frequency bands}

One can clearly see that in this case the damage feature completely fails in identifying the true location of damage using the arbitrarily chosen frequency, namely $10-100 \mathrm{~Hz}$ and $10-30 \mathrm{~Hz}$. (Figs 32 and 33).

The fact that these results give unacceptable values for the damage indicator was expected, considering that in these arbitrary chosen frequency bands the differences in the transmissibility functions are more influenced by the changing loading condition then by the change of the dynamic properties caused by the damage.

\subsubsection{Using small frequency bands around the resonance frequencies}

As was suggested by the theory and the simulations we are now going to have a look around a small frequency band around the resonance frequencies of the structure. This careful selection of the frequency band should result in a more reliable damage indication in the case of changing loading conditions. 

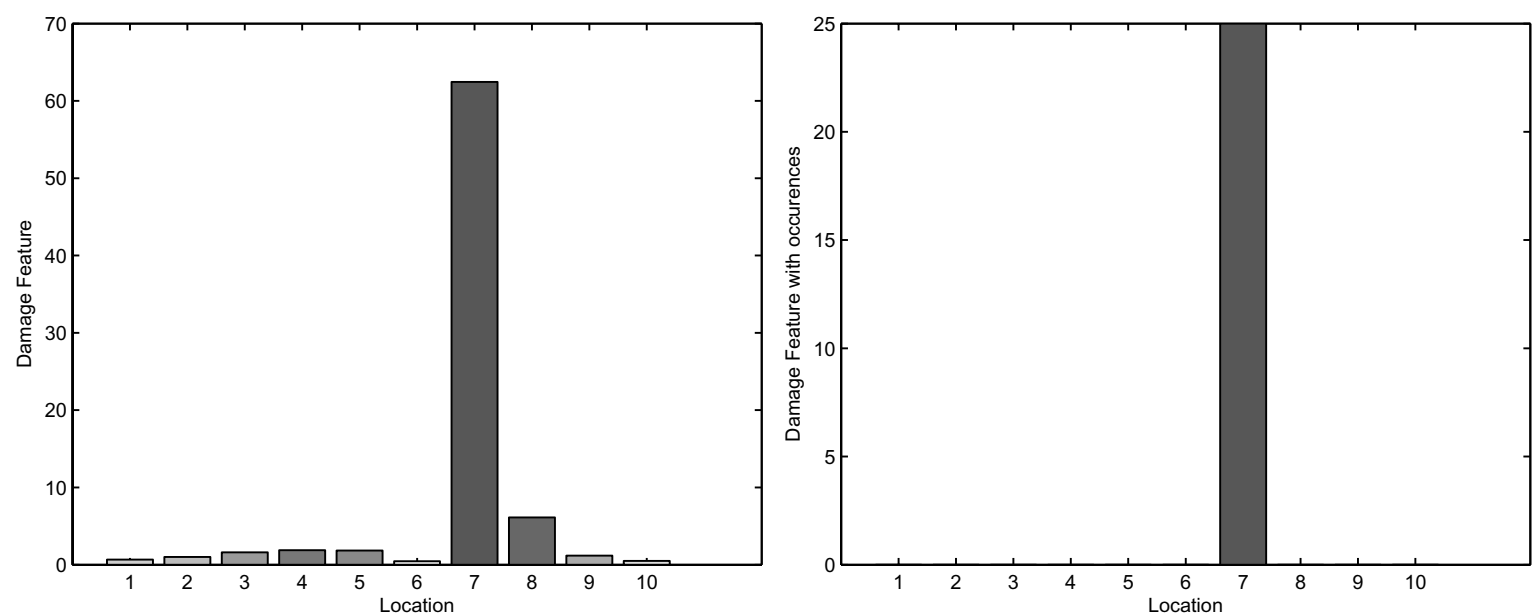

Fig. 34. Damage feature without and with occurrences for damaged structure and changing loading conditions, frequency range $83-86 \mathrm{~Hz}$.
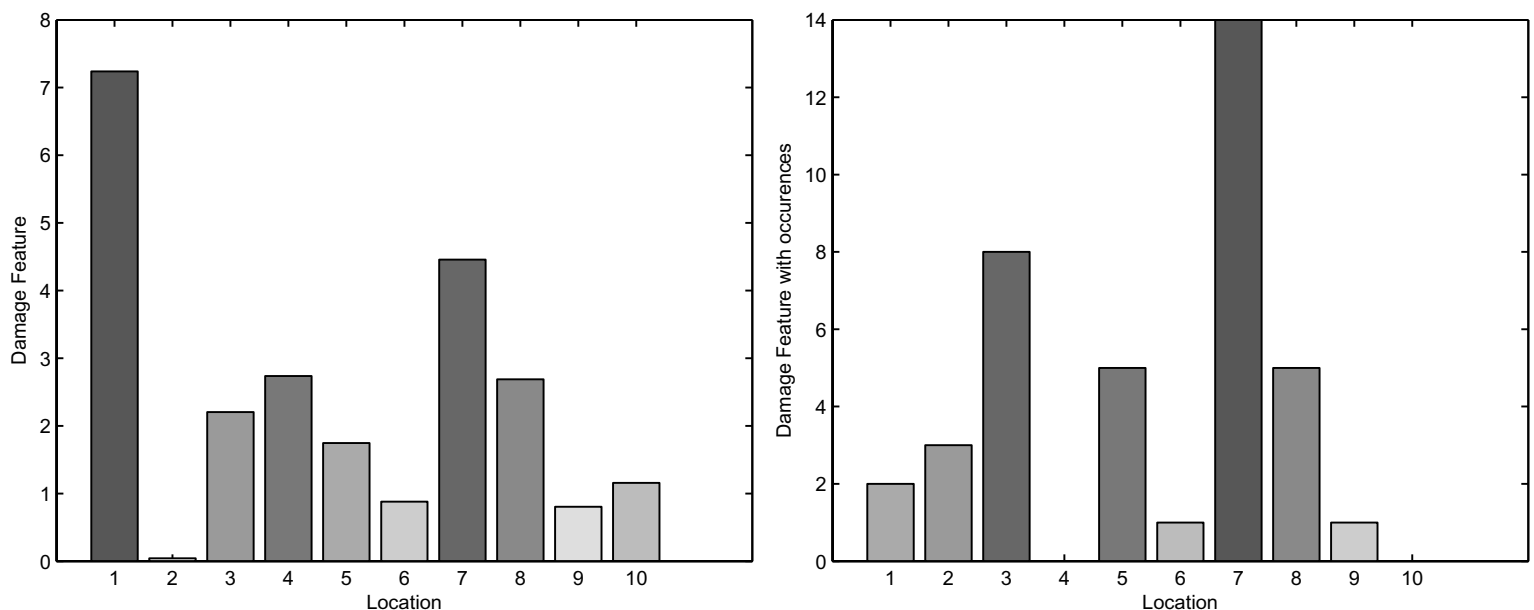

Fig. 35. Damage feature without and with occurrences for damaged structure and changing loading conditions, frequency range 10-15Hz.
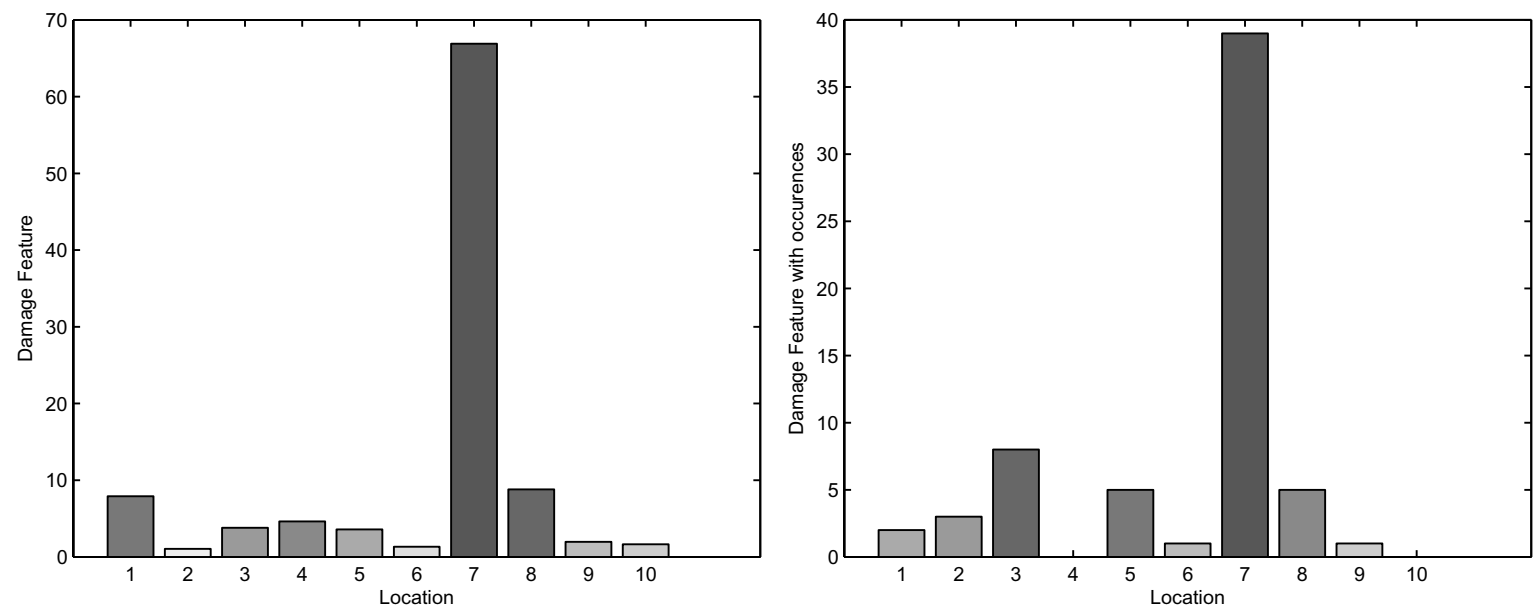

Fig. 36. Damage feature without and with occurrences for damaged structure and changing loading conditions with frequency weighting. 

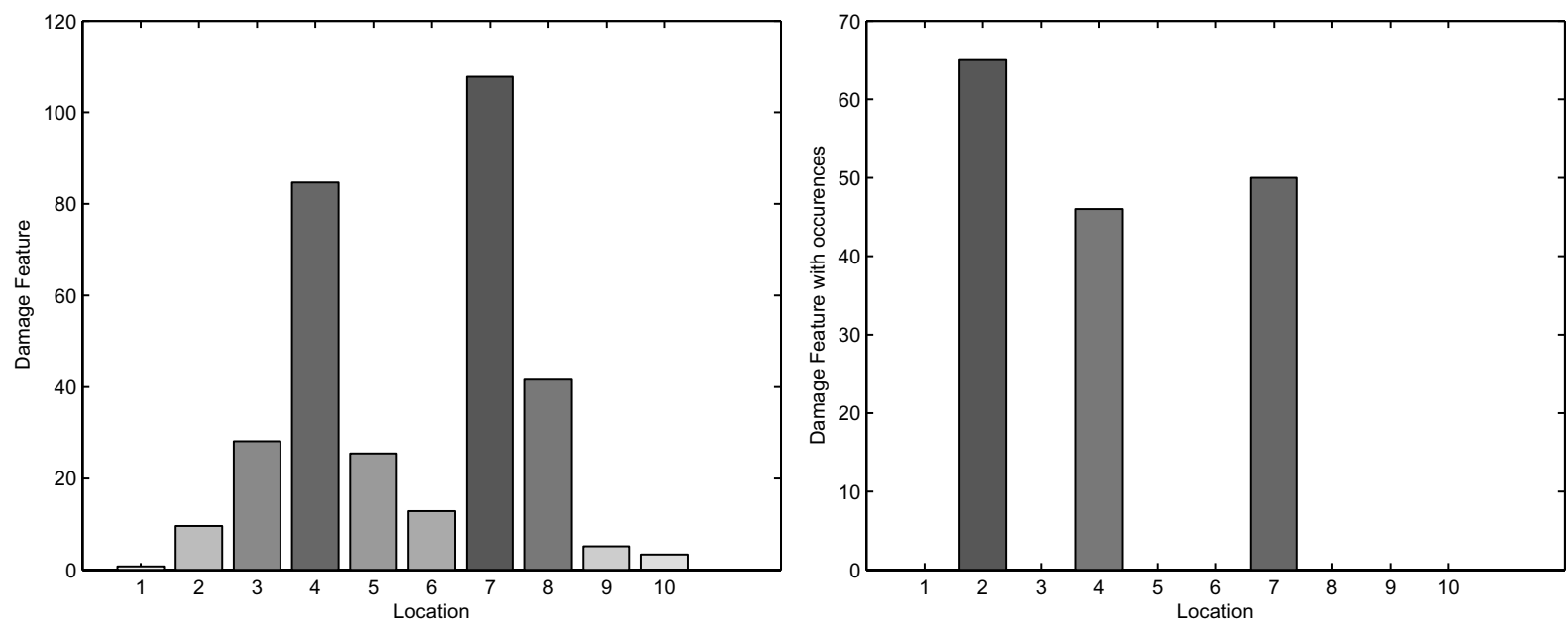

Fig. 37. Damage feature without and with occurrences for damaged structure and changing loading conditions, frequency range 75-95Hz.

When using a small frequency band around the second resonance frequency the damage feature perfectly indicates the correct location of the damage, for both approaches without and with occurrences (See Fig. 34). In a small frequency band around the first resonance frequency the results are less clear for this experiment (See Fig. 35).

So far all presented damage indicators use a uniform weighted linear sum of all spectral lines of an arbitrary chosen frequency range. In practice in the case of changing loading conditions a possible reliable approach would be to use a carefully chosen weighting, where we use only the information in small bands around the resonance frequencies of the structure and discard all other information.

In Fig. 36 you can see the obtained results for such an approach. It gives the damage feature with frequency weighting if we combine the information of only the two previously selected frequency bands, $10-15 \mathrm{~Hz}$ and $83-$ $86 \mathrm{~Hz}$, respectively around the first and second resonance frequency of the structure. One can easily identify the correct damage location. This approach is assumed to result in a reliable damage indicator using transmissibility functions in changing loading conditions.

\subsubsection{Using bigger frequency bands around the resonance frequencies}

Figure 37 shows the damage feature calculated using a bigger frequency band around the second resonance frequency. As expected the results deteriorate, as more non-zero values of the damage feature appear again at other locations then the damage location. The damage indicator becomes again unreliable.

The same effect on the damage feature can be found by using a bigger frequency band around the first resonance frequency (Fig. 31).

\subsubsection{Using small frequency bands not around the resonance frequencies}

Also if we select a frequency band not around the resonance frequencies the results become completely unreliable (Figs 38 and 39).

In these frequency bands the change in transmissibilities is almost completely influenced by the change in loading conditions and almost not affected by the damage. This could already be observed in Fig. 29. As a result a lot of non-zero values of the damage feature appear at random locations different from the correct damage location.

\section{Final remarks}

In practice, even in the case of constant loading conditions, one might consider to use only the information in small bands around the resonance frequencies of the structure and discard all other information. Figure 40 shows the results for the damage feature, for a constant loading condition, with the proposed frequency weighting. For this 

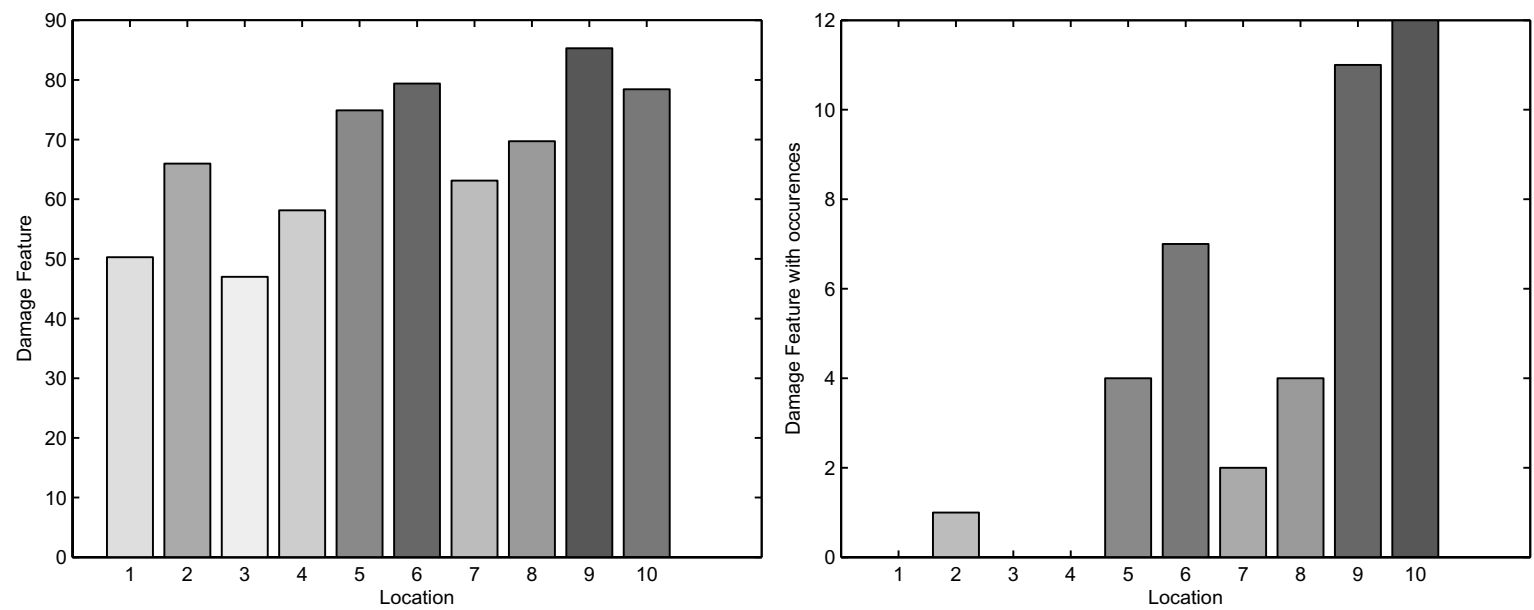

Fig. 38. Damage feature without and with occurrences for damaged structure and changing loading conditions, frequency range $25-30 \mathrm{~Hz}$.
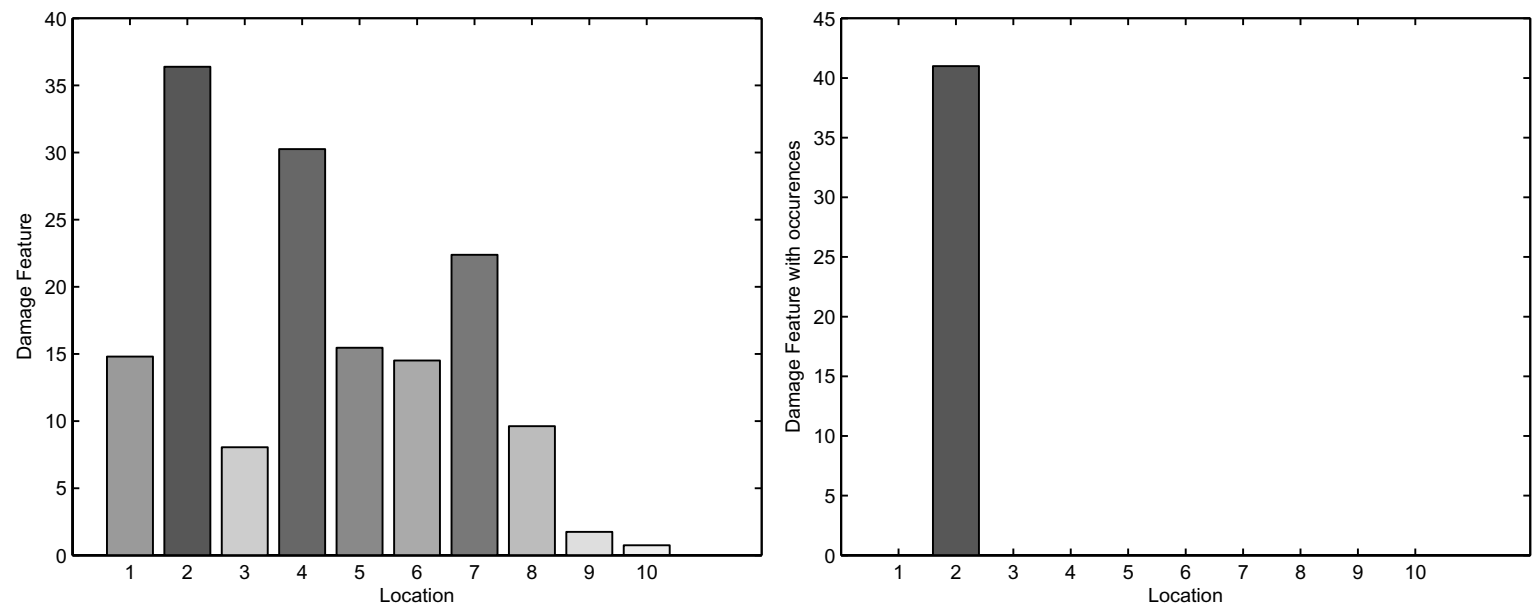

Fig. 39. Damage feature without and with occurrences for damaged structure and changing loading conditions, frequency range 90-95 Hz.
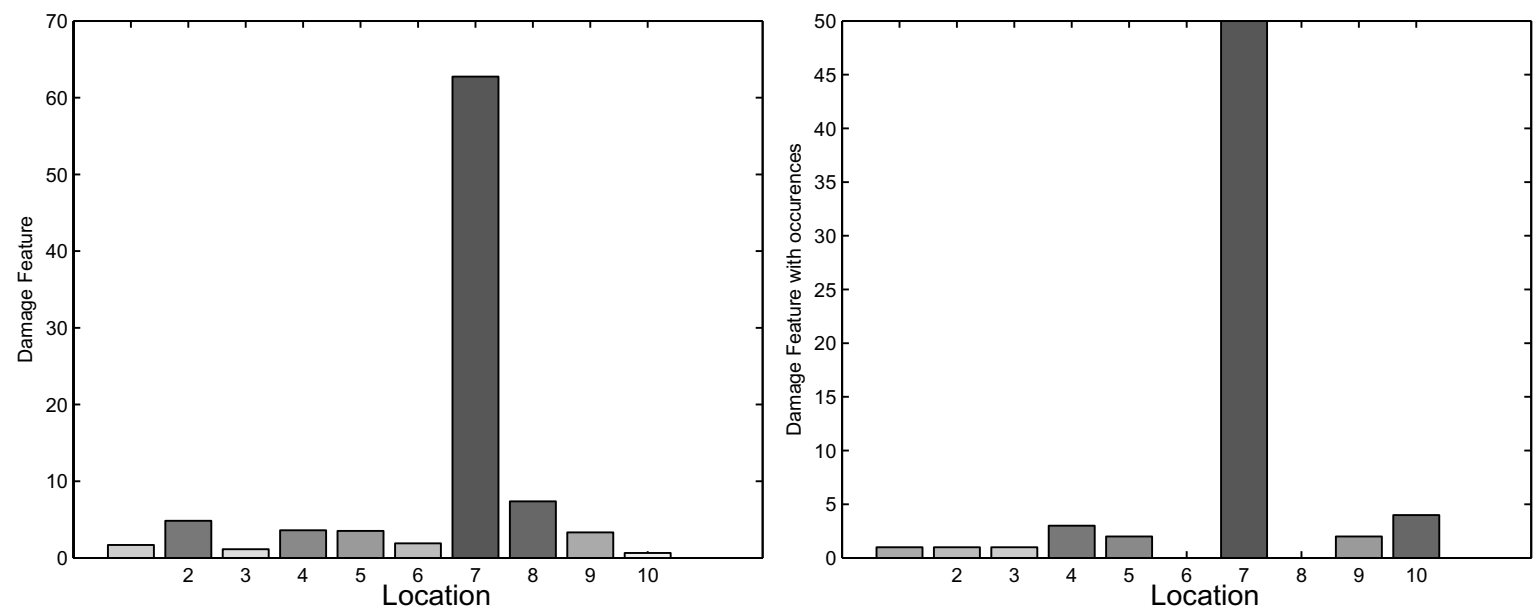

Fig. 40. Damage feature without and with occurrences for damaged structure and constant loading conditions with frequency weighting. 

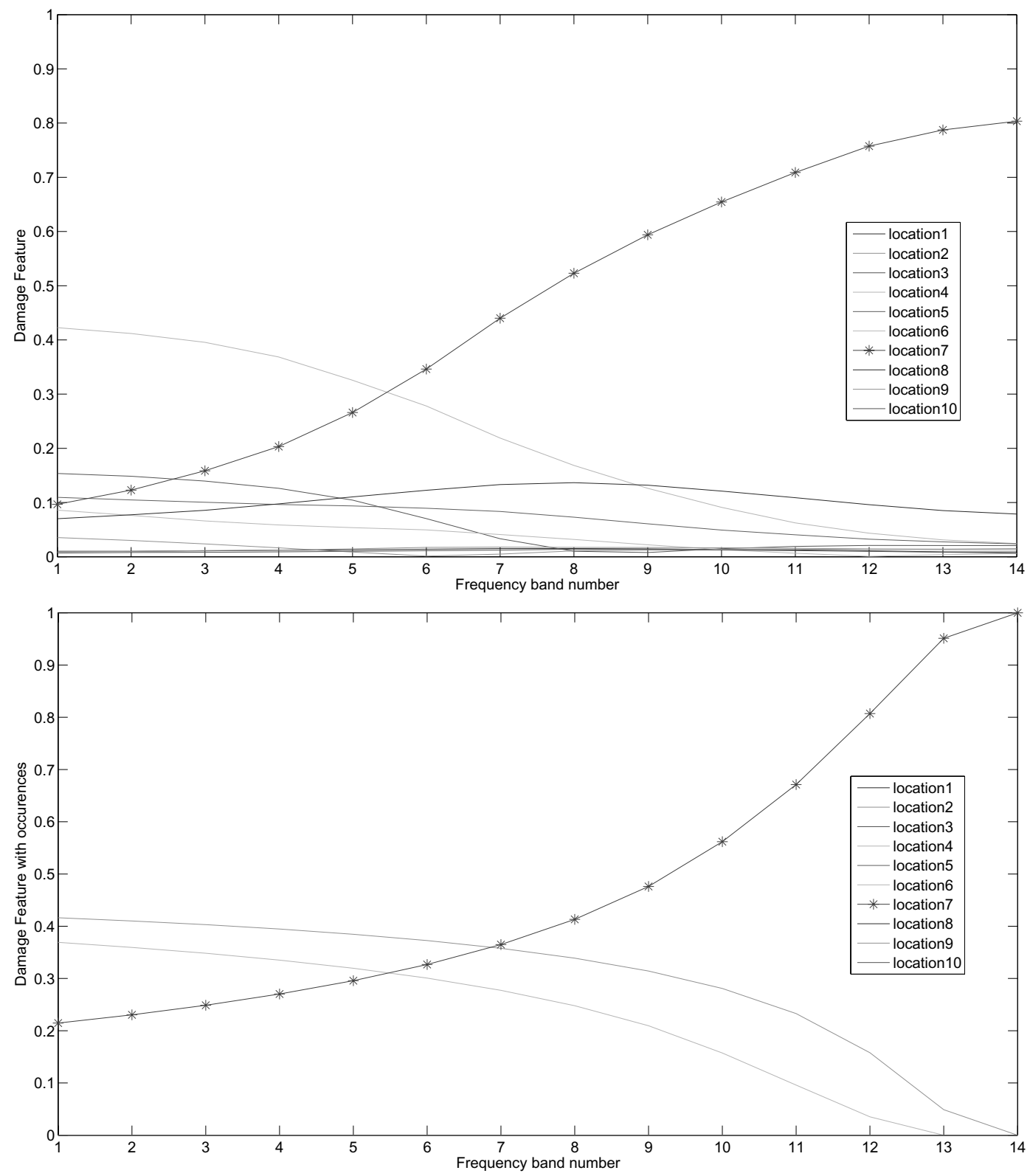

Fig. 41. Relative damage feature without (top) and with occurrences (bottom) for damaged structure and changing loading conditions for different frequency bands.

case we only used the information of the frequency bands, $10-15 \mathrm{~Hz}$ and $83-86 \mathrm{~Hz}$, respectively around the first and second resonance frequency of the structure, and discarded all other information. At first glance the results are easier to analyze compared to those obtained using the arbitrary frequency band of $10-100 \mathrm{~Hz}$ or $10-30 \mathrm{~Hz}$, as was shown in Figs 30 and 31.

It was suggested in this paper to use only the information coming from sufficient small frequency bands around the resonance frequencies of the structure. It is clear that this sufficient small has not yet properly been defined in this paper and that it is part of future work. However one can understand that this will depend on the level of change in the operational loading conditions and the level of change of the health condition of the structure and their relative importance on the transmissibility functions. 
In practice one might also think to not only have a look to the value of the damage feature in one frequency band but track the relative changes of the damage feature over different bands that become e.g. smaller and smaller around the resonance frequencies of the structure. The relative value of the damage feature at the different locations could be used as an indicator for the correct location of the damage.

The result of such an exercise can be seen in Fig. 41. Fourteen successive smaller frequency bands around the second resonance frequency have been taken, respectively 70-99 HZ, 71-98 Hz, 72-97 Hz . . 82-87 Hz and $83-86 \mathrm{~Hz}$. The value of the damage feature is the relative value per frequency band. One can observe that the relative value of the damage feature for location 7 (full line with marker*) is increasing with smaller frequency bands, while the relative values of the damage feature at the locations without damage is decreasing. This observation can directly be used to correctly locate the damage.

\section{Conclusions}

This paper has shown that changing loading conditions do not automatically exclude the use of existing damage indicators based on transmissibility measurements. Caution has to be taken in the selection of the used frequency band in order to eliminate false damage notifications.

It has been shown that under varying loading conditions, when taking small frequency bands around the resonance frequencies of the structure, accurate results on damage location can be found. Therefore it was suggested to use a proper frequency weighting, where we use only the information in small bands around the resonance frequencies of the structure and discard all other information, in order to calculate the damage feature.

Finally is has been shown that tracking the relative changes of the damage feature over different frequency bands, that become smaller and smaller around the resonance frequencies of the structure, can also be used to correctly locate the damage.

\section{Acknowledgements}

This research has been supported by the Institute for the Promotion of Innovation by Science and Technology in Flanders (IWT), the Fund for Scientific Research-Flanders (Belgium) (FWO) and by the Research Council (OZR) of the Vrije Universiteit Brussel (VUB).

\section{References}

[1] S.W. Doebling, C.R. Farrar, M.B. Prime and D.W. Shevitz, Damage Identification and Health Monitoring of Structural and Mechanical Systems from Changes in Their Vibration Characteristics: A Literature Review., Los Alamos National Laboratory Report, LA-13070-MS, 1996.

[2] C.R. Farrar, S.W. Doebling and T.A. Duffer, Vibration-based Damage Detection, in: Structural Dynamics @ 2000: Current Status and Future Directions, D.J. Ewins and D.J. Inman, eds, Baldock; Hertfortshire, England: Research Studies Press Ltd, 2001, pp. 145-174.

[3] R.P.C. Sampaio, N.M.M. Maia and J.M.M. Silva, Damage detection using the frequency-response-function curvature method, Journal of Sound and Vibration 226(5) (Oct 1999), 1029-1042.

[4] N.M.M. Maia, J.M.M. Silva, E.A.M. Almas and R.P.C. Sampaio, Damage Detection in Structures: from Mode Shape to Frequency Response Function Methods, Mechanical Systems and Signal Processing 17(Issue 3) (2002), 489-498.

[5] N.M.M. Maia, R.P.C. Sampaio, A.M.R. Ribeiro and J.M.M. Silva, Transmissibility Techniques for Damage Detection? Proc. of IMAC XIX, Kissimmee, 2001.

[6] T.J. Johnson and D.E. Adams, Transmissibility as a Differential Indicator of Structural Damage, J Vib Acoust 124(Issue 4) (October 2002), 634.

[7] C. Devriendt and P. Guillaume, Identification of modal parameters from transmissibility measurements? Journal of Sound and Vibration 314(Issue 1-2) (2007), 343-356.

[8] T.J. Johnson, Analysis of Dynamic Transmissibility as a Future for Structural Damage Detection, MSc. Thesis, Purdue University, 2002.

[9] Vanlanduit, Steve; Daerden, Frank; Guillaume Patrick; Experimental modal testing using pressurized air excitation, Journal of Sound and Vibration 1-2 (2006), 83-98. 

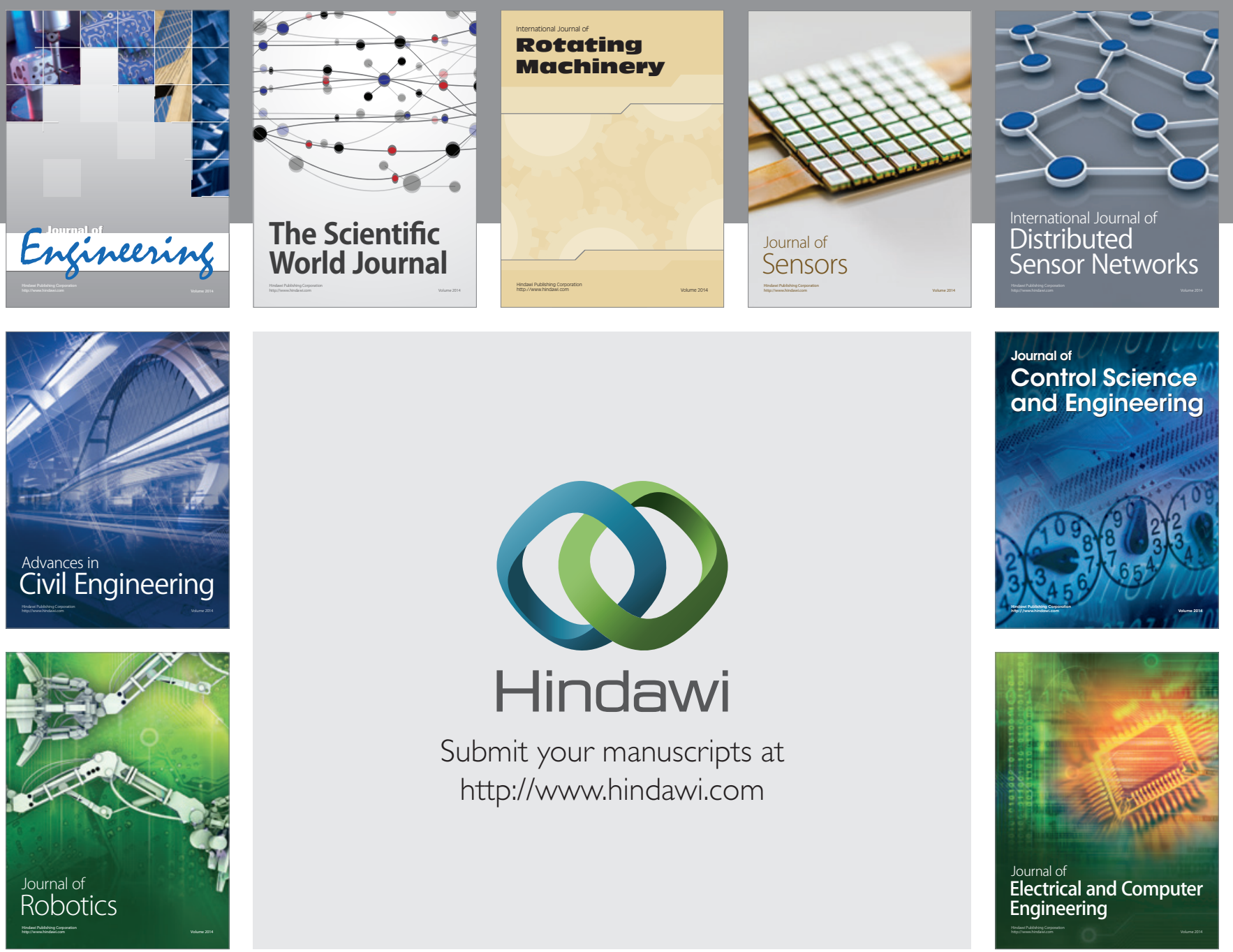

Submit your manuscripts at

http://www.hindawi.com
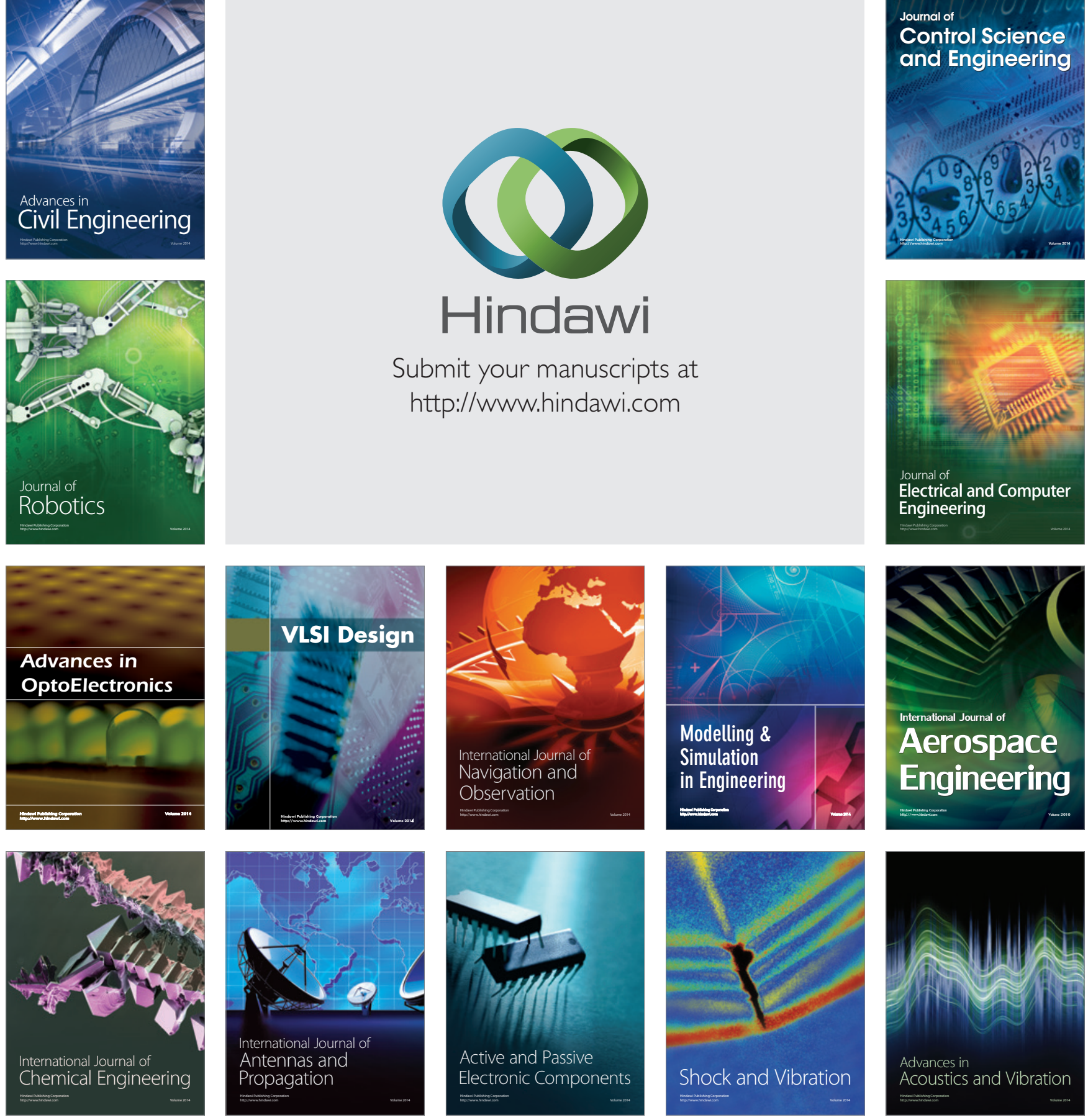